\title{
Comparing Libraries for Generic Programming in Haskell
}

Alexey Rodriguez

Johan Jeuring

Patrik Jansson

Alex Gerdes

Oleg Kiselyov

Bruno C. d. S. Oliveira

Department of Information and Computing Sciences, Utrecht University

Technical Report UU-CS-2008-010

www.cs.uu.nl

ISSN: 0924-3275 


\section{Comparing Libraries for Generic Programming in Haskell}

\author{
Alexey Rodriguez \\ Utrecht University, The Netherlands \\ alexey@cs.uu.nl
}

\section{Alex Gerdes}

Open University, The Netherlands alex.gerdes@ou.nl

\author{
Johan Jeuring \\ Utrecht University, The Netherlands \\ johanj@cs.uu.nl
}

\author{
Oleg Kiselyov \\ FNMOC, USA \\ oleg@pobox.com
}

\author{
Patrik Jansson \\ Chalmers University of Technology \& \\ University of Gothenburg, Sweden \\ patrikj@chalmers.se
}

\author{
Bruno C. d. S. Oliveira \\ Oxford University, UK \\ bruno.oliveira@comlab.ox.ac.uk
}

\begin{abstract}
Datatype-generic programming is defining functions that depend on the structure, or "shape", of datatypes. It has been around for more than 10 years, and a lot of progress has been made, in particular in the lazy functional programming language Haskell. There are more than 10 proposals for generic programming libraries or language extensions for Haskell. To compare and characterize the many generic programming libraries in a typed functional language, we introduce a set of criteria and develop a generic programming benchmark: a set of characteristic examples testing various facets of datatype-generic programming. We have implemented the benchmark for nine existing Haskell generic programming libraries and present the evaluation of the libraries. The comparison is useful for reaching a common standard for generic programming, but also for a programmer who has to choose a particular approach for datatype-generic programming.
\end{abstract}

\section{Introduction}

Software development often consists of designing a datatype to which functionality is added. Some functionality is datatype specific. Other functionality is defined on almost all datatypes, and only depends on the structure of the datatype; this is called datatype-generic functionality. Examples of datatype-generic functionality are comparing two values for equality, searching a value of a datatype for occurrences of a particular string or other value, editing a value, pretty-printing a value, etc. Larger examples include XML tools, testing frameworks, debuggers, and data-conversion tools.

Datatype-generic programming has been around for more than 10 years now. A lot of progress has been made in the last decade, in particular with generic programming in the lazy functional programming language Haskell. There are more than 10 proposals for generic programming libraries or language extensions for Haskell. Such libraries and extensions are also starting to appear for other programming languages, such as ML.

Although generic programming has been used in several applications, it has few users for real-life projects. This is understandable. Developing a large application takes a couple of years, and choosing a particular approach to generic programming for such a project involves a risk. Few approaches that have been developed over the last decade are still supported, and there is a high risk that the chosen approach will not be supported anymore, or that it will change in a backwards-incompatible way in a couple of years time.
The Haskell Refactorer HaRe [Li et al., 2003] is an exception, and provides an example of a real-life project in which a genericprogramming technique (Strafunski [Lämmel and Visser, 2002]) is used to implement traversals over a large abstract syntax tree. However, this project contains several other components that could have been implemented using generic-programming techniques, such as rewriting, unification, and pretty-printing modules. These components are much harder to implement than traversals over abstractsyntax trees. Had these components been implemented generically, we claim that, for example, the recent work of the HaRe team to adapt the refactoring framework to the Erlang language [Derrick and Thompson, 2005] would have been easier. Other exceptions are the Haskell application Server (HappS), which uses the extensible variant of Scrap Your Boilerplate [Lämmel and Peyton Jones, 2005], and the Catch [Mitchell and Runciman, 2007a] and Reach [Naylor and Runciman, 2007] tools, which use the Uniplate [Mitchell and Runciman, 2007b] library to implement traversals.

It is often not immediately clear which generic programming approach is best suited for a particular project. There are generic functions that are difficult or impossible to define in certain approaches. The set of datatypes to which a generic function can be applied varies among different approaches, and the amount of work a programmer has to do per datatype and/or generic function varies as well.

The current status of generic programming in Haskell is comparable to the lazy Tower of Babel preceding the birth of Haskell in the eighties [Hudak et al., 2007]. We have many single-site languages or libraries, each individually lacking critical mass in terms of language/library-design effort, implementations, and users.

How can we decrease the risk in using generic programming? We propose to design a common generic programming library for Haskell. To increase the chances of continuing support, we will develop this library in an international committee. The rationale for developing a library for generic programming instead of a language extension is that Haskell is powerful enough to support most generic programming concepts by means of a library. Furthermore, compared with a language extension, a library is much easier to ship, support, and maintain. The library might be accompanied by tools that depend on non-standard language extensions, for example for generating embedding-projection pairs, as long as the core is standard Haskell. The standard that the library design should target is Haskell 98 and widely-accepted extensions (such as existential types and multi-parameter type classes) that are likely to be included in the next Haskell standard [Haskell Prime list, 2006]. 
The library should support the most common generic programming scenarios, so that programmers can define the generic functions that they want and use them with the datatypes they want.

To design a common generic programming library, we first have to evaluate existing libraries to find out differences and commonalities, and to be able to make well-motivated decisions about including and excluding features. This paper is about comparing existing libraries for generic programming in Haskell. We will evaluate and compare the following libraries:

- Lightweight Implementation of Generics and Dynamics (LIGD) [Cheney and Hinze, 2002]

- Polytypic programming in Haskell (PolyLib) [Norell and Jansson, 2004]

- Scrap your boilerplate (SYB) [Lämmel and Peyton Jones, 2003, 2004]

- Scrap your boilerplate, extensible variant using typeclasses [Lämmel and Peyton Jones, 2005]

- Scrap your boilerplate, spine view variant [Hinze et al., 2006, Hinze and Löh, 2006]

- Generics for the Masses (GM) [Hinze, 2006], including its extensible and modular extension (EMGM) [Oliveira et al., 2006].

- RepLib: a library for derivable type classes [Weirich, 2006]

- Smash your boilerplate [Kiselyov, 2006]

- Uniplate [Mitchell and Runciman, 2007b]

Note that this list does not contain generic programming language extensions such as PolyP [Jansson and Jeuring, 1997], Generic Haskell [Löh et al., 2003], or Template Haskell [Lynagh, 2003], and no pre-processing approaches to generic programming such as DrIFT [Winstanley and Meacham, 2006], and Data.Derive. We strictly limit ourselves to library approaches, which, however, might be based on particular compiler extensions. The SYB [Lämmel and Peyton Jones, 2005] and Strafunski [Lämmel and Visser, 2003] approaches are very similar, and therefore we only take the SYB approach into account in this evaluation. The functionality of the Compos library [Bringert and Ranta, 2006] is subsumed by Uniplate, and hence we only evaluate the latter.

We evaluate existing libraries by means of a set of criteria. Papers about generic programming usually give desirable criteria for generic programs. Examples of such criteria are: can a generic function be extended with special behaviour on a particular datatype, and are generic functions first-class, that is, can they take a generic function as argument. We develop a set of criteria based on our own ideas about generic programming, and ideas from papers about generic programming. For most criteria, we have a generic function that determines whether or not the criterion is satisfied. We have collected a set of generic functions for testing the criteria. We try to implement all of these functions in the different approaches.

We are aware of two existing comparisons of support for generic programming in programming languages. Garcia et al. [2007] compare the support for different kinds of generic programming in a number of programming languages. Haskell supports all their eight criteria. We use more fine-grained criteria to distinguish the Haskell libraries which support datatype-generic programming. Hinze et al. [2007] compare various approaches to datatype-generic programming in Haskell. However, most of the covered approaches are language extensions, and many of the recent library approaches have not been included.

This paper has the following contributions:
- It gives an extensive set of criteria for comparing libraries for generic programming in Haskell. The criteria might be viewed as a characterisation of generic programming in Haskell.

- It develops a generic programming benchmark: a set of characteristic examples with which we can test the criteria for generic programming libraries.

- It compares nine existing library approaches to generic programming in Haskell with respect to the criteria, using the implementation of the benchmark in the different libraries.

- The benchmark itself is a contribution. It can be seen as a cookbook that illustrates how different generic programming tasks are achieved using the different approaches. Furthermore, its availability makes it easier to compare the expressiveness of future generic programming libraries. The benchmark suite can be obtained following the instructions at http://haskell. org/haskellwiki/GPBench.

The outcome of this evaluation is not necessarily restricted to the context of Haskell. We think this comparison will be relevant for other programming languages as well.

This paper is organised as follows. Section 2 introduces datatypegeneric programming concepts and terminology. Section 3 shows the design and contents of the benchmark suite. Section 4 introduces and discusses the criteria we use for comparing libraries for generic programming in Haskell. Section 5 summarizes the evaluation of the different libraries with respect to the criteria, using the benchmark. Section 6 presents the evaluation in full detail. Section 7 concludes.

\section{Generic programming: concepts and terminology}

This section introduces and illustrates generic programming using a simplified form of the datatype-generic programming library LIGD. The reason for using LIGD is that the encoding mechanisms of this library are simple and probably easier to understand than those of other more advanced libraries. We refer the reader to the original LIGD paper [Cheney and Hinze, 2002] for a more detailed explanation of this approach.

In polymorphic lambda calculus it is impossible to write one parametrically polymorphic equality function that works on all datatypes [Wadler, 1989]. That is why the definition of equality in Haskell uses type classes, and ML uses equality types. The $E q$ type class provides the equality operator $==$, which is overloaded for a family of types. To add a newly defined datatype to this family, the programmer defines an instance of equality for it. Thus, the programmer must manually write definitions of equality for every new datatype that is defined. For equality, this process could be automated by using the type class deriving mechanism. However, this mechanism can only be used with a small number of type classes because it is hardwired into the language, making it closed and impossible to extend or change by the programmer.

Generic programming addresses the concern mentioned above in the form of generic functions. Generic functions are defined once and work for a large family of datatypes. In generic programming, the introduction of a new datatype does not require redefinition or extension of all existing generic functions. We merely need to describe the new datatype to the library, and all existing and future generic functions will be able to handle it.

Below we give a brief introduction to generic programming and the terminology that we use throughout this paper.

A type-indexed function (TIF) is a function that is defined on every type of a family of types. We say that the types in this family index the TIF, and we call the type family a universe. A TIF is defined by case analysis on types: each type is assigned a 


$$
\begin{aligned}
& \text { geq }:: \text { Rep a } \rightarrow \mathrm{a} \rightarrow \mathrm{a} \rightarrow \text { Bool } \\
& \text { geq (RUnit) Unit Unit }=\text { True }
\end{aligned}
$$

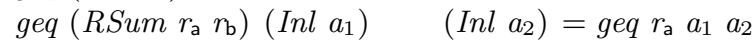

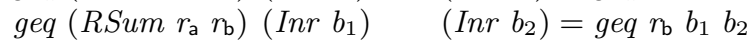

$$
\begin{aligned}
& \text { geq }\left(\text { RSum } r_{\mathrm{a}} r_{\mathrm{b}}\right)_{-} \quad-\quad=\text { False } \\
& \text { geq }\left(\operatorname{RProd} r_{\mathrm{a}} r_{\mathrm{b}}\right)\left(\operatorname{Prod} a_{1} b_{1}\right)\left(\operatorname{Prod} a_{2} b_{2}\right) \\
& =g e q r_{\mathrm{a}} a_{1} a_{2} \wedge g e q r_{\mathrm{b}} b_{1} b_{2}
\end{aligned}
$$

Figure 1. Type-indexed equality function in the LIGD library

$$
\begin{aligned}
& \text { data Unit }=\text { Unit } \\
& \text { data Sum } \mathrm{a} \mathrm{b}=\operatorname{Inl} \mathrm{a} \mid \operatorname{Inr} \mathrm{b} \\
& \text { data Prod } \mathrm{a} \mathrm{b}=\operatorname{Prod} \mathrm{a} \mathrm{b}
\end{aligned}
$$

Figure 2. Unit, sum and product datatypes

function that acts on values of that type. As a familiar example, consider the TIF equality implemented using Haskell type classes. The universe consists of the types that are instances of the $E q$ type class. Equality for each of these types is given by the == method of the corresponding instance. And the case analysis on types is provided by instance selection.

Haskell type classes are only one of the possible implementations of TIFs. In this section we use LIGD with Generalised Algebraic Datatypes (GADTs) [Peyton Jones et al., 2006] to implement TIFs. As an example, we start with the equality TIF which is indexed by a universe consisting of units, sums and products. We show the definitions in Figures 1 and 2. Note that in Haskell type variables appearing in type signatures are implicitly universally quantified.

The first argument of the function is a type representation, it describes the type of the values that are to be compared (second and third arguments). Haskell does not allow functions to depend on types, so here types are represented by a GADT. This has the advantage that case analysis on types can be implemented by pattern matching, a familiar construct to functional programmers. The GADT represents the types of the universe consisting of units, sums and products:

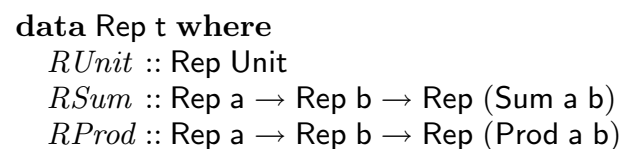

geq has three type-indexed function cases, one for each of the base types of the universe.

Let us now turn to the concept of TIF instantiation. Instantiation is the process by which we make a TIF specific to some type t, so that we can apply the resulting function to $t$ values. In LIGD the instantiation process is straightforward: $g e q$ performs a fold over Rep $t$ using pattern matching, and builds an equality function that can be used on $t$ values. In other approaches, instantiation uses, for example, the type class system.

Suppose now that we want to instantiate equality to lists. Since a generic function can only be instantiated to types inside the universe, we need to extend our universe to lists. There are two ways to do this. The first is non-generic extension, we extend our case analysis on types so that lists are handled by equality. In LIGD, this translates into the following: extend Rep with an RList constructor that represents lists, and extend equality with a case for RList:

$$
\text { geq }\left(\text { RList } r_{\mathrm{a}}\right) \text { xs ys }=\ldots
$$

The second way to implement extension is generic extension: we describe the structure of the list datatype in terms of types inside the universe. The consequence is that instantiation to lists does not need a special case for lists, but reuses the existing cases for sums, products and units. To make the idea more concrete let us have a look at how type structure is represented in LIGD.

In LIGD, the structure of a datatype $b$ is represented by the following Rep constructor.

$$
\text { RType }:: \operatorname{Rep~c~} \rightarrow \text { EP b c } \rightarrow \text { Rep b }
$$

The type $c$ is the structure representation type of $b$, where $c$ is a type isomorphic to $b$. The isomorphism is witnessed by an embedding projection pair, which is a pair of functions that convert $b$ values to $c$ values and back.

$$
\text { data EP b c }=E P\{\text { from }::(\mathrm{b} \rightarrow \mathrm{c}), \text { to }::(\mathrm{c} \rightarrow \mathrm{b})\}
$$

In LIGD, constructors are represented by nested sum types and constructor arguments are represented by nested product types. The structure representation type for lists is Sum Unit (Prod a [a]), and the embedding projection for lists is as follows:

$$
\begin{aligned}
& \text { fromList :: [a] } \rightarrow \text { Sum Unit (Prod a [a]) } \\
& \text { fromList [] = Inl Unit } \\
& \text { fromList }(a: a s) \quad=\operatorname{Inr}(\operatorname{Prod} a \text { as }) \\
& \text { toList :: Sum Unit (Prod a [a]) } \rightarrow[\mathrm{a}] \\
& \text { toList (Inl Unit) }=\text { [] } \\
& \text { toList }(\operatorname{Inr}(\text { Prod a as }))=a \text { : as }
\end{aligned}
$$

To extend the universe to lists, we write a type representation using RType:

$$
\begin{aligned}
r \text { List } \quad:: \text { Rep a } \rightarrow & \text { Rep }[\mathrm{a}] \\
\text { rList } r_{\mathrm{a}}=\text { RType } & \left(\text { RSum RUnit }\left(\text { RProd } r_{\mathrm{a}}\left(\text { rList } r_{\mathrm{a}}\right)\right)\right) \\
& (\text { EP fromList toList })
\end{aligned}
$$

Generic equality is still missing a case to handle datatypes that are represented by RType. The definition of this case is given below. It takes two values, transforms them to their structure representations and recursively applies equality.

$$
\text { geq (RType } \left.r_{\mathrm{a}} \text { ep) } t_{1} t_{2}=\text { geq } r_{\mathrm{a}}\left(\text { from ep } t_{1}\right) \text { (from ep } t_{2}\right)
$$

In summary, there are two ways to extend a universe to a type $T$. Non-generic extension requires type-specific, ad-hoc cases for $\mathrm{T}$ in type-indexed functions, and generic-extension requires a structure representation of $T$ but no additional function cases. This is a distinguishing feature between type-indexed functions and generic functions. The latter include a case for RType, which allows them to exploit the structure of a datatype in order to apply generic uniform behaviour to values of that datatype; while the former do not have a case for RType, and therefore rely exclusively on nongeneric extension.

In LIGD, sums, products, and units are used to represent the structure of a datatype. Certainly, other choices are possible. For example, PolyLib includes the datatype Fix in the universe, in order to represent the recursive structure of datatypes. We refer to these representation choices as generic views [Holdermans et al., 2006]. Informally, a view consists of base (or view) types for the universe (for example Sum and Prod) and a convention to represent structure, for example, the fact that constructors are represented by nested sums. The choice of a view will often impact the expressiveness of a library, that is, which generic function definitions are supported and what are the set of datatypes on which generic extension is possible.

\section{Design of the benchmark suite}

Most previous work on datatype-generic programming focuses on either increasing the number of scenarios in which generic pro- 
gramming can be applied, or on obtaining the same number of scenarios using fewer or no programming language extensions. For example, Hinze's work on "Polytypic values possess polykinded types" [Hinze, 2002] shows how to define generic functions that work on types of arbitrary kinds, instead of on types of a particular kind, and "Generics for the Masses" [Hinze, 2006] shows how to do a lot of generic programming without using Haskell extensions. Both goals are achieved by either inventing a new generic programming approach altogether, or by extending an existing approach.

We have collected a number of typical generic programming scenarios from the literature. These are used as a guide to design our benchmark suite. The intuition is that the evaluation of a library should give an accurate idea of how well the library supports the generic programming scenarios. We list the scenarios below:

- Generic versions of Haskell type class functionality such as equality $(E q)$, comparison (Ord) and enumeration (Enum) [Jansson and Jeuring, 1998].

- Serialisation and deserialisation functions such as read and show in Haskell [Jansson and Jeuring, 2002].

- Traversals to query and modify information in datatypes [Lämmel and Peyton Jones, 2003].

- Functions like map, crush, and transpose, which manipulate elements of a parametrised datatype such as lists [Jansson and Jeuring, 1998, Norell and Jansson, 2004].

- Data conversion [Jansson and Jeuring, 2002, Atanassow and Jeuring, 2004].

- Test data generation [Koopman et al., 2003, Lämmel and Peyton Jones, 2005].

We have identified the features that are needed from a generic library to implement the scenarios above. These features are used as criteria to characterise generic programming from a user's point of view, where a user is a programmer who writes generic programs. There are also users who only use generic programs (such as people that use deriving in Haskell), but the set of features needed by the latter kind of users is a subset of that needed by the former. Generic programming scenarios are not the only source of criteria, we also use the following sources:

- new features introduced by existing approaches such as Hinze [2002],

- Comparing approaches to generic programming in Haskell [Hinze et al., 2007],

- the Haskell generics wiki page [Haskell Generic Library list, 2008],

- our own ideas, based on several years experience with different approaches to generic programming.

We test whether the criteria are fulfilled with a benchmark suite. Each function in the suite tests whether or not an approach satisfies a particular criterion. For example, generic map cannot be implemented if the "abstraction over type constructors" criterion is not supported by the library. Hence, if a library cannot be used to implement a function, it means that it does not support the criterion that the function is testing. Each function in the benchmark suite can be regarded as a simplified version of one of the above programming scenarios.

Before introducing the functions used in the benchmark suite, we describe the datatypes on which they are used, and the related structure representation machinery.

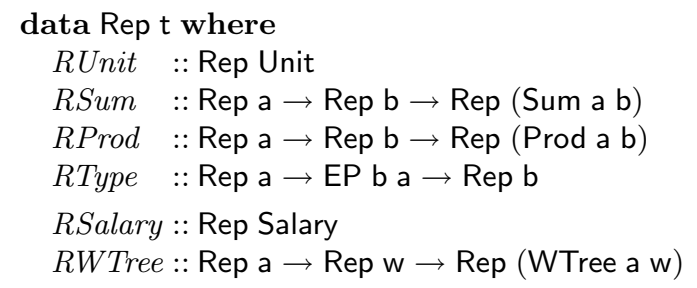

Figure 3. Definition of Rep. The two last constructors are not part of the LIGD library.

$$
\begin{array}{ll}
r \text { Company } & :: \operatorname{Rep} \text { Company } \\
r \text { Dept } & :: \operatorname{Rep} \text { Dept } \\
r \text { BinTree } & :: \operatorname{Rep} \mathrm{a} \rightarrow \operatorname{Rep}(\text { BinTree a) } \\
r \text { WTree } & :: \operatorname{Rep} \mathrm{a} \rightarrow \operatorname{Rep} \mathrm{w} \rightarrow \operatorname{Rep}(\text { WTree a w) } \\
r \text { Rose } & ::(\forall \mathrm{a} \cdot \operatorname{Rep} \mathrm{a} \rightarrow \operatorname{Rep}(\mathrm{f} \text { a) } \rightarrow \\
& \operatorname{Rep} \mathrm{a} \rightarrow \operatorname{Rep}(\text { Gose } \mathrm{f} \text { a) }
\end{array}
$$

Figure 4. Type signatures of some type representations.

\subsection{Datatypes}

The datatype construct in Haskell combines many aspects: type abstraction and application, recursion, records, local polymorphism, etc. In this section we introduce a number of datatypes, that cover many of these aspects. A generic programming library that can apply generic functions to one of these datatypes is said to support the aspects that the datatype requires in its definition.

The aspects that we test for are: parametrised types (type constructors, which use type abstraction and type application), both simple and nested recursion, higher-kinded datatypes (which have a parameter of kind $\star \rightarrow \star$ ) and constructor name information (in order to implement generic show).

The aspects that we do not test in this paper are higher-rank constructors (explicit forall in the datatype declaration), existential types, GADTs, and parsing related information, namely record label names, constructor fixity, and precedence. The three first aspects are not tested because they are hardly supported by any of the libraries that we evaluate. The last aspect, parsing-related information, can be incorporated using the same mechanisms as for providing constructor names, and therefore we do not add datatypes that test for this aspect.

Following each datatype definition we must also provide the machinery that allows universe extension for the particular library we are using. For LIGD each datatype T must map to a structure representation type $\mathrm{T}^{\prime}$ and back, with functions from $T$ and to $T$. The type representation for $\mathrm{T}$ is $r T$, it is written using RType like the list representation ( $r$ List) in Section 2. However, two of the datatypes presented below, namely Salary and WTree, are used in non-generic extension tests. For this reason the definition of Rep in Figure 3 includes the constructors RSalary and RWTree.

The company datatype. The Company datatype represents the organisational structure of a company. It was introduced in the first SYB paper.

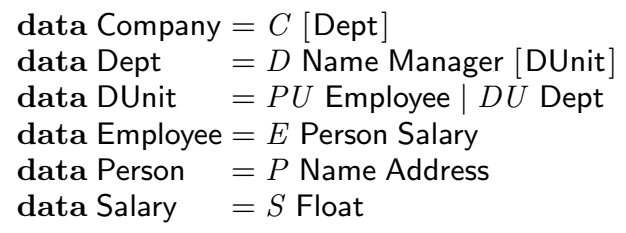




$$
\begin{array}{ll}
\text { type Manager } & =\text { Employee } \\
\text { type Name } & =\text { String } \\
\text { type Address } & =\text { String }
\end{array}
$$

To define the representation of Company we must also define the representation of the supporting datatype Dept. The same holds for the other company datatypes.

$$
\begin{aligned}
& r \text { Company }=\text { RType }(r \text { List } r \text { Dept }) \\
& \quad(\text { EP fromCompany toCompany }) \\
& r \text { Dept }=\ldots
\end{aligned}
$$

Because Salary is used with non-generic extension, the representation uses RSalary directly:

$$
r \text { Salary }=\text { RSalary }
$$

Binary trees. The recursive BinTree datatype abstracts over the type of its elements stored in the leaves.

$$
\text { data BinTree a = Leaf a } \mid \text { Bin (BinTree a) (BinTree a) }
$$

Like lists, the representation of a binary tree is a function on representations that depends on the representation of a:

$$
\begin{gathered}
r \text { BinTree } r_{\mathrm{a}}=\text { let } r=\text { rBinTree } r_{\mathrm{a}} \text { in } \\
\text { RType }\left(\text { RSum } r_{\mathrm{a}}(\text { RProd } r \mathrm{r})\right) \\
(\text { EP fromBinTree toBinTree })
\end{gathered}
$$

Trees with weights. We adapt the type of binary trees such that we can assign a weight, whose type is abstracted, to a (sub)tree.

$$
\begin{aligned}
\text { data WTree a } \mathrm{w}= & \text { WLeaf a } \\
\mid & \text { WBin (WTree a w) (WTree a w) } \\
\mid & \text { WithWeight (WTree a w) w }
\end{aligned}
$$

Some of the generic function tests treat weights differently from elements, even if their types are the same.

The representation of WTree and the remaining datatypes are omitted because they follow they same pattern as rBinTree defined just above. However, Section 3.2.3 uses a different structure representation of WTree to define specialised behaviour for constructors.

Generalised rose trees. Rose trees are (non-empty) trees whose internal nodes have a list of children instead of just two.

$$
\text { data Rose a }=\text { Node a [Rose a] }
$$

We can generalise Rose by abstracting from the list datatype:

$$
\text { data GRose } \mathrm{f} a=\text { GNode a ( } \mathrm{f} \text { (GRose } \mathrm{f} \mathrm{a}) \text { ) }
$$

The interesting aspect that GRose tests is higher-kindedness: it takes a type constructor argument $\mathrm{f}$ of kind $\star \rightarrow \star$.

Perfect trees. The datatype Perfect is used to model perfect binary trees: binary trees that have exactly $2^{n}$ elements, where $n$ is the depth of the binary tree.

$$
\begin{aligned}
& \text { data Perfect a }=\text { Zero a } \mid \text { Succ (Perfect (Fork a)) } \\
& \text { data Fork a }=\text { Fork a a }
\end{aligned}
$$

The depth of a perfect binary tree is the Peano number represented by its constructors. The datatype Perfect is a so-called nested datatype [Bird and Meertens, 1998], because the type argument changes from a to Fork $\mathrm{a}$ in the recursion.

Nested generalised rose trees. The NGRose datatype is a variation on GRose that combines nesting with higher-kinded arguments: at every recursive call $\mathrm{f}$ is passed composed with itself:

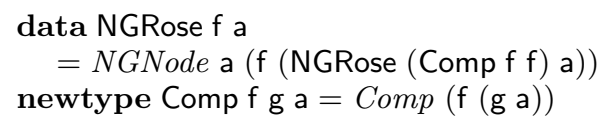

Non-generic representations for Salary and WTree. Two of the datatypes introduced above are used in tests that check whether a library supports non-generic extension. Because non-generic extension is not supported by LIGD, we assume that Rep includes representation constructors for those datatypes in order to be able to describe the extension tests. The full definition of Rep and the signatures of some representations are shown in Figures 3 and 4. Note that post-hoc addition of constructors to the Rep datatype is a suboptimal idea that will break existing code. Concretely, the definition of $g e q$ in this paper is for the first four constructors (RUnit, $R S u m$, RProd, RType) of Rep, thus any use of geq on RSalary or $R W$ Tree will fail. We return to this problem in Section 5 where we discuss support for ad-hoc definitions in LIGD.

\subsection{Functions}

Inspired by the generic programming scenarios given at the beginning of this section, we describe a number of generic functions for our benchmark suite.

It is not necessary to include all functions arising from the generic programming scenarios. If two functions use the same set of features from a generic programming library, it follows that if one of them can be implemented, the other can be implemented too. For example, the test case generator, generic read, and generic enumeration functions rely on library support for writing producer functions. So, it is enough to test that feature with only one function, and hence we omit the last two functions from the benchmark suite.

\subsubsection{Generic variants of type class functionality: Equality}

Generic equality takes a type representation argument Rep a and produces the equality function for a-values.

$$
\text { geq }:: \operatorname{Rep} \mathrm{a} \rightarrow \mathrm{a} \rightarrow \mathrm{a} \rightarrow \text { Bool }
$$

On most datatypes we assume that two values are equal if and only if they have the same constructor and if the arguments of the constructors are pairwise equal. In LIGD, constructors are encoded as nested sum types. It follows that two values are equal only if they have the same constructor (Inl or Inr) and the constructor arguments are equal too. The constructor arguments are encoded as nested products, hence product equality must require the equality of corresponding components. The implementation in Fig. 1 shows the sum and product cases. Constructor names are ignored - only positions of constructors in the "constructor list" and positions of arguments in the "argument list" are taken into account.

The generic version of the Ord method, compare, would have type $\operatorname{Rep} \mathrm{a} \rightarrow \mathrm{a} \rightarrow \mathrm{a} \rightarrow$ Ordering. Like equality it takes two arguments and consumes them (as opposed to producing a value of that type). Approaches that can implement equality can also implement comparison.

\subsubsection{Serialisation and deserialisation: Show}

The show function takes a value of a datatype as input and returns its representation as a string. It can be viewed as the implementation of deriving Show in Haskell. Its type is as follows:

$$
\text { gshow }:: \text { Rep a } \rightarrow \text { a } \rightarrow \text { String }
$$

The function gshow is used to test the ability of generic libraries to provide constructor names for arbitrary datatypes. For the sake of simplicity this function is not a full replacement of Haskell's show:

- The generic show function treats lists in the same way as other algebraic datatypes. (Note that in the examples that follow we use $\rightsquigarrow$ to indicate reductions of expressions.)

$$
\text { gshow }[1,2] \rightsquigarrow "(:) 1 \text { ((:) } 2[]) "
$$


Note, however, that gshow is extended in one of the tests to print lists using Haskell notation. This is a separate test that is called gshowExt.

- It also treats strings just as lists of characters:

$$
\text { gshow "GH" } \rightsquigarrow "(:) \text { 'G' ((:) 'H' [] )" }
$$

- Other features that are not supported are constructor fixity, precedence and record labels.

\subsubsection{Querying and transformation traversals}

A typical use of generic functions is to collect all occurrences of elements of a particular constant type in a datatype. For example, we might want to collect all Salary values that appear in a datatype:

$$
\text { selectSalary }:: \text { Rep a } \rightarrow \text { a } \rightarrow \text { [Salary }]
$$

We can instantiate this function to Company:

$$
\text { selectSalary rCompany :: Company } \rightarrow \text { [Salary] }
$$

Collecting values is an instance of a more general pattern: querying traversals. The function above can be implemented using (1) a general function (which happens to be generic) that performs the traversal of a datatype, and (2) a specific case that actually collects the Salary values.

Querying traversals are interesting because they require two features from a generic programming library:

- The ability to define a generic function that has an ad-hoc (nonuniform) definition for some type. For example, salaryCase returns a singleton list of its argument if applied to a Salary value. Otherwise it returns the empty list.

$$
\begin{aligned}
& \text { salaryCase }:: \text { Rep a } \rightarrow \text { a } \rightarrow[\text { Salary }] \\
& \text { salaryCase RSalary sal }=[\text { sal }] \\
& \text { salaryCase rep - }=[]
\end{aligned}
$$

The LIGD library does not support this feature, but we extended the Rep type in Fig. 3 to be able to show what it would look like.

- The ability to define a generic function that takes another generic function as argument. Consider for example (the LIGD version of) the $g$ map $Q$ function from the first SYB paper,

$$
\text { gmap } Q::(\forall \mathrm{a} . \operatorname{Rep} \mathrm{a} \rightarrow \mathrm{a} \rightarrow \mathrm{r}) \rightarrow \operatorname{Rep} \mathrm{b} \rightarrow \mathrm{b} \rightarrow[\mathrm{r}]
$$

such that

$$
\text { gmapQ } f r T\left(\begin{array}{llll}
K & a_{1} & \ldots & a_{n}
\end{array}\right) \rightsquigarrow\left[\begin{array}{lrl}
f & r T_{1} & a_{1}, \ldots, f r T_{n} a_{n}
\end{array}\right]
$$

This function takes three arguments: a generic function $f$, a type representation and a value of that type. If the value is a constructor $K$ applied to a number of arguments, gmap $Q$ returns a list of $f$ applied to each of the arguments.

Using salaryCase as argument it gives:

$$
\begin{aligned}
\text { gmap } Q \text { salaryCase }(\text { rList rSalary }) & \left(S 1.0:\left[\begin{array}{ll}
S & 2.0
\end{array}\right]\right) \\
\rightsquigarrow & {[\text { salaryCase rSalary } \quad(S 1.0)} \\
& , \text { salaryCase }(r \text { List rSalary })\left[\begin{array}{ll}
S & 2.0
\end{array}\right] \\
\rightsquigarrow & {\left[\left[\begin{array}{ll}
S & 1.0
\end{array}\right],[]\right] }
\end{aligned}
$$

It is not a good idea to test for both features with one single test case in our suite: if a library does not support one of them the other will remain untested. For this reason we test these two features separately, using the functions selectSalary and gmap $Q$ :

$$
\begin{aligned}
& \text { selectSalary }:: \text { Rep } \mathrm{a} \rightarrow \mathrm{a} \rightarrow[\text { Salary }] \\
& \operatorname{gmap} Q::(\forall \mathrm{a} . \operatorname{Rep} \mathrm{a} \rightarrow \mathrm{a} \rightarrow \mathrm{r}) \rightarrow \operatorname{Rep} \mathrm{a} \rightarrow \mathrm{a} \rightarrow[\mathrm{r}]
\end{aligned}
$$

Transformation traversals. An obvious variation on queries are transformation traversals. A typical example of such a traversal consists of transforming some nodes while performing a bottomup traversal. Function updateSalary increases all occurrences of Salary by some factor in a value of an arbitrary datatype.

$$
\begin{aligned}
& \text { updateSalary }:: \text { Float } \rightarrow \text { Rep a } \rightarrow \mathrm{a} \rightarrow \mathrm{a} \\
& \text { updateSalary } 0.1 \text { (rList rSalary) }[S \text { 1000.0, } S \text { 2000.0 }] \\
& \quad \rightsquigarrow[S 1100.0,2200.0]
\end{aligned}
$$

Transformations on constructors. The updateSalary function traverses datatypes other than Salary generically, in other words the traversal is performed on the structure representation using the cases for products, sums and units. It follows that it is unnecessary to supply ad-hoc traversal cases for such datatypes.

In updateSalary, the ad-hoc behaviour targets a particular datatype. Constructor cases [Clarke and Löh, 2003] are a refinement of this idea. They let us introduce ad-hoc behaviour that instead targets a particular constructor, and handles the rest generically.

Suppose that we want to apply the optimisation rule

$$
x+0 \rightarrow x
$$

to values of a datatype which consists of a large number of constructors. Ideally we would like constructors other than sum to be traversed generically, and only an ad-hoc case for sums, implementing the rewrite rule.

The benchmark suite includes the function rmWeights to test ad-hoc behaviour for constructors. This function removes the weight constructors from a WTree

$$
\begin{gathered}
\text { rmWeights (RWTree RInt RInt) } \\
\text { (WBin (WithWeight (WLeaf 42) 1) } \\
\text { (WithWeight (WLeaf 88) 2)) } \\
\rightsquigarrow(\text { WBin (WLeaf 42) (WLeaf 88)) }
\end{gathered}
$$

The definition of the transformation handles the With Weight constructor and lets the remaining constructors be handled by the generic machinery.

$$
\begin{aligned}
& r m \text { Weights }:: \text { Rep a } \rightarrow \text { a } \rightarrow \text { a } \\
& r m \text { Weights } r @(\text { RTree ra rw) } t= \\
& \text { case } t \text { of } \\
& \quad \text { WithWeight } t^{\prime} w \rightarrow r m \text { Weights } r t^{\prime} \\
& \quad t^{\prime} \quad \begin{aligned}
\rightarrow \\
\text {... handle generically ... }
\end{aligned} \\
& \text { rest of definition omitted ... }
\end{aligned}
$$

The second arm of the case traverses the structure representation of $t^{\prime}$ generically rather than matching WBin and WLeaf explicitly. The full code of the function is shown in Fig. 5.

The last line of the definition uses $r W$ Tree to traverse the structure representation of $t^{\prime}$. Because it is essential that remaining WithWeight constructors in $t^{\prime}$ are removed, the definition of $r W$ Tree has to be altered for this function. The recursive occurrences of WTree have to be represented by $R W T$ Tree rather than $r W T r e e$ as is usually done in other structure representations. In this way traversals of the subtrees will again be handled by the adhoc case (see Fig. 5).

\subsubsection{Abstraction over type constructors: crush and map}

The function crushRight [Meertens, 1996] is a generic fold-like function. Typical instances are summing all integers in a list, or flattening a tree into a list of elements.

$$
\begin{aligned}
& \text { sumList }::[\operatorname{lnt}] \rightarrow \operatorname{lnt} \\
& \text { sumList }[2,3,5,7] \rightsquigarrow 17 \\
& \text { flattenBinTree }:: \text { BinTree } \mathrm{a} \rightarrow[\mathrm{a}]
\end{aligned}
$$




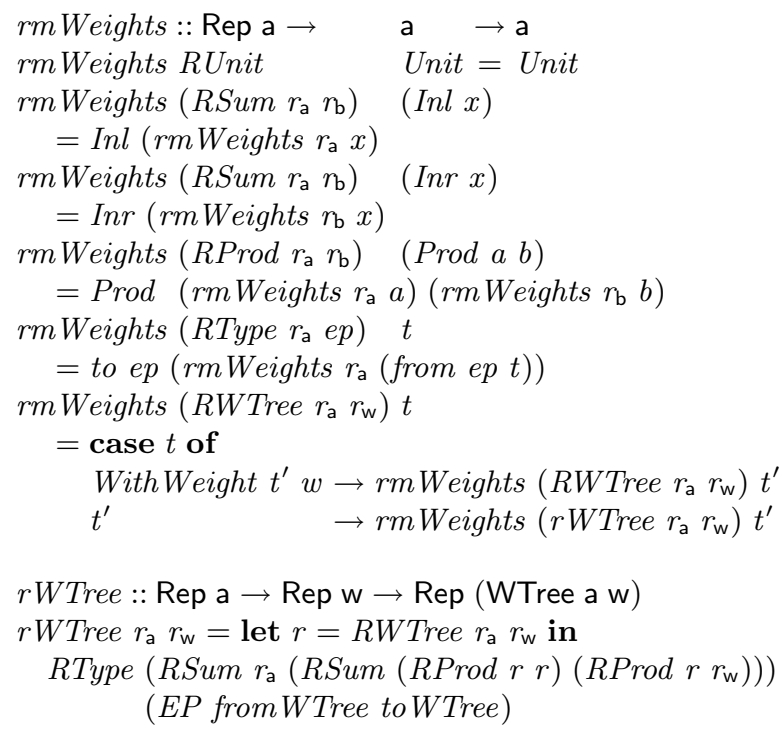

Figure 5. Generically remove weights from a WTree.

\section{flattenBinTree (Bin (Leaf 2) (Leaf 1)) $\rightsquigarrow[2,1]$}

The generic version of these functions abstracts over the type of the structure:

$$
\text { crushRight }:: \operatorname{Rep}^{\prime} \mathrm{f} \rightarrow(\mathrm{a} \rightarrow \mathrm{b} \rightarrow \mathrm{b}) \rightarrow \mathrm{f} \mathrm{a} \rightarrow \mathrm{b} \rightarrow \mathrm{b}
$$

The function crushRight traverses the $f$ a structure right to left accumulating a value of type $b$, which is updated by combining it with every a-value that is encountered during the traversal.

So far, generic functions have been using a type representation that encodes types of kind $\star$. Lists are not an exception: $r$ List $r_{\mathrm{a}}$ represents fully applied list types. In order to define crushRight we switch to a type representation that encodes types of kind $\star \rightarrow \star$. This is why we use Rep' instead of Rep (and below $r$ List' instead of $r$ List). This is a common situation: to increase expressiveness of a generic library the type representation is adjusted. This is also an unfortunate situation because, in general, different type and structure representations are mutually incompatible.

Function crushRight can be instantiated on lists or trees, and given the right arguments, we obtain the definitions of sumList and flattenBin Tree:

$$
\begin{aligned}
& \text { sumList } \quad x s=\text { crushRight rList }{ }^{\prime}(+) \text { xs } 0 \\
& \text { flattenBinTree bt }=\text { crushRight } r \text { Tree }{ }^{\prime}(:) \text { bt [] }
\end{aligned}
$$

How are generic queries different from crushRight? We could for example define a function selectInt to flatten a BinTree Int into a list of Int values. There are two differences. First, if the BinTree elements were booleans instead of integers, we would need a different querying function: selectBool. With flattenBin Tree we do not have these problems because it is parametrically polymorphic in the elements of the datatype.

The second difference is about the type signature of the querying function. Suppose now that we want to flatten WTree Int Int into a list of weights.

$$
\begin{aligned}
& \text { flatten WTWeights :: WTree a w } \rightarrow[\mathrm{w}] \\
& \text { flattenWTWeights }(\text { WBin }(\text { WithWeight (WLeaf 1) 2) } \\
& \quad \text { (WithWeight (WLeaf 3) 4)) } \\
& \qquad[2,4]
\end{aligned}
$$

This is just an instance of crushRight:

\section{flatten WTWeights tree $=$ crushRight $r W$ Tree $^{\prime}($ :) tree []}

where $r$ WTree' represents WTree a for any a. With queries, it is not at all obvious how to implement this. For selectInt there is no difference between Int-weights and Int-elements in the tree. So it gives the following incorrect result:

$$
\begin{aligned}
& \text { selectInt (WBin }(\text { WithWeight (WLeaf 1) 2) } \\
& \quad(\text { WithWeight }(\text { WLeaf } 3) 4)) \\
& \rightsquigarrow[1,2,3,4]
\end{aligned}
$$

To sum up, the difference with queries is that crushRight views the datatype as an application of a type constructor $f$ to an element type $a$, and processes only a-values. In contrast, traversal queries do not make such element discrimination based on the type structure of the datatype.

Map. Generic map is to transformation traversals what crushRight is to query traversals. The gmap function takes a function and a structure of elements, and applies the function argument to all elements in that structure. The type signature of gmap uses the same representation as crushRight:

$$
\text { gmap }:: \operatorname{Rep}^{\prime} \mathrm{f} \rightarrow(\mathrm{a} \rightarrow \mathrm{b}) \rightarrow \mathrm{f} \mathrm{a} \rightarrow \mathrm{f} \mathrm{b}
$$

The best known instance is the map function on lists, but we also have instances like

$$
\text { gmap rBinTree }::(\mathrm{a} \rightarrow \mathrm{b}) \rightarrow \text { BinTree } \mathrm{a} \rightarrow \text { BinTree } \mathrm{b}
$$

In general, gmap can be viewed as the implementation of deriving for the Functor type class in Haskell.

Another function, generic transpose, is representative not only of abstraction over type constructors but also of data conversion functions. We discuss it next.

\subsection{Data conversion: transpose}

Data conversion functions have type $T \rightarrow T^{\prime}$ : they convert $T$ values into $T^{\prime}$ values. Generic approaches to build conversion functions are discussed in the work of Jansson and Jeuring [2002] and Atanassow and Jeuring [2004]. It turns out that there is no need to include the conversion functions from these sources, because the conversion functions are built out of simpler generic functions which are already accounted for in our scenarios. The former paper uses a combination of serialisation, deserialisation, and abstraction over type constructors. The latter paper composes serialisation and deserialisation functions that exploit isomorphisms in the intermediate structures.

A more sophisticated version of data conversion is the generic transpose function, described in Norell and Jansson [2004]. Although this function can be implemented using a combination of serialisation, deserialisation, and abstraction over type constructors, it is an interesting challenge for library approaches because it abstracts over two type constructors.

The generic transpose function is a generalisation of the function transpose that is defined in the Haskell standard library. Since this function abstracts over two type constructors it takes two type representations:

$$
\text { gtranspose }:: \operatorname{Rep}^{\prime} \mathrm{f} \rightarrow \operatorname{Rep}^{\prime} \mathrm{g} \rightarrow \mathrm{f}(\mathrm{g} \text { a }) \rightarrow \mathrm{g}(\mathrm{f} \text { a })
$$

Instantiating both $\mathrm{f}$ and $\mathrm{g}$ on the list datatype we obtain transpose again.

$$
\begin{aligned}
& \text { transpose }=\text { gtranspose } r \text { List }^{\prime} \text { rList }^{\prime} \\
& \text { transpose }[[1,2,3],[4,5,6]] \rightsquigarrow[[1,4],[2,5],[3,6]]
\end{aligned}
$$

It can also be instantiated to other datatypes:

$$
\begin{aligned}
& \text { gtranspose rList }{ }^{\prime} \text { rBinTree' }[\text { Leaf } 1, \text { Leaf 2, Leaf 3] } \\
& \quad \rightsquigarrow \text { Leaf }[1,2,3]
\end{aligned}
$$




\subsubsection{Test data generation: Fulltree}

Testing is used to gain confidence in a program. QuickCheck [Claessen and Hughes, 2000] is a popular tool that supports automatic testing of properties of programs. A user-defined datatype can be used in an automated test, provided it is an instance of the Arbitrary class.

To implement the test data generation scenario, a library should be able to produce values. The test data generation scenario is represented by the gfulltree function.

The gfulltree function takes a representation of a container datatype as input and returns a value of the represented datatype. An additional integer parameter is used to constrain the size of the value. The actual size of the value depends on the type itself and its representation. In the case of LIGD and a binary tree, the size determines the depth of the tree. Here are the type signature of gfulltree and some examples of its usage:

$$
\begin{aligned}
& \text { gfulltree }:: \text { Rep a } \rightarrow \operatorname{Int} \rightarrow \mathrm{a} \\
& \text { gfulltree }(\text { rList rInt) } 6 \rightsquigarrow[0,0,0,0,0,0] \\
& \text { gfulltree }(\text { rBinTree rInt) } 2 \\
& \quad \rightsquigarrow \operatorname{Bin}(\text { Bin (Leaf } 0)(\text { Leaf 0)) (Bin (Leaf 0) (Leaf 0)) }
\end{aligned}
$$

Function gfulltree can also be used to generate large values that are used in performance tests.

\subsubsection{More general representations of type constructors}

The generic functions above use type representations over types of kind $\star$ :

$$
\text { geq }:: \operatorname{Rep} \mathrm{a} \rightarrow \mathrm{a} \rightarrow \mathrm{a} \rightarrow \text { Bool }
$$

and over types of kind $\star \rightarrow \star$

$$
\text { gmap }:: \operatorname{Rep}^{\prime} \mathrm{f} \rightarrow(\mathrm{a} \rightarrow \mathrm{b}) \rightarrow \mathrm{f} \mathrm{a} \rightarrow \mathrm{f} \mathrm{b}
$$

What if we want to apply gmap to transform both the payload and weights of a WTree? Should we define Rep" to represent types of kind $\star \rightarrow \star \rightarrow \star$ ? It is not good practice to define a new representation every time we come across a kind that was not previously representable.

Below we discuss briefly how some libraries generalise over types of arbitrary kinds. While those solutions are more general (and maybe more useful) than having one representation per kind, the full discussion of these solutions would complicate the presentation. We continue to use Rep and Rep' in this paper to keep the presentation of gmap and crushRight simple.

Representations and arities. The work of Hinze [2002] introduces a technique to allow generic functions to be applied to type constructors of arbitrary kinds. The technique consists in generalizing the type signature of a generic function so that it becomes generic in more than one type variable. Let us examine this technique in a library setting, here we use the representations introduced by the GM approach [Hinze, 2006].

The idea is that GM type representations, in addition to abstract over a type a:

$$
\text { data } \operatorname{Rep} a=\ldots
$$

they also abstract over the signature of a generic function:

$$
\text { data } \operatorname{Rep} 1(\operatorname{sig}:: \star \rightarrow \star)(\mathrm{a}:: \star)=\ldots
$$

Here sig could be used, for example, with the signature of equality (newtype $\mathrm{Eq} \mathrm{a}=E q(\mathrm{a} \rightarrow \mathrm{a} \rightarrow$ Bool)) or generic show (newtype Show a $=$ Show $(\mathrm{a} \rightarrow$ String)). Generic map, however, cannot be defined using Rep1 because it needs to change the type of its argument. So the type signature needs to abstract over two types rather than one: newtype Map a b $=\operatorname{Map}(\mathrm{a} \rightarrow \mathrm{b})$. This prompts for a new representation:

$$
\begin{aligned}
\text { data Rep2 } & \text { sig a b where } \\
\text { RUnit2 } & :: \text { Rep2 sig Unit Unit } \\
\text { RSum2 } & :: \text { Rep2 sig a b } \rightarrow \text { Rep2 sig c d } \\
& \rightarrow \text { Rep2 sig (Sum a c) (Sum b d) } \\
\text { RProd2 } & :: \text { Rep2 sig a b } \rightarrow \text { Rep2 sig c d } \\
& \rightarrow \text { Rep2 sig (Prod a c) (Prod b d) } \\
\text { RType2 } & :: \text { Rep2 sig a d } \rightarrow \text { EP b a } \rightarrow \text { EP e d } \\
& \rightarrow \text { Rep2 sig b e } \\
\text { RVar2 } & :: \text { sig a b } \rightarrow \text { Rep2 sig a b } \\
r \text { Tree2 } & :: \text { Rep2 sig a b } \rightarrow \text { Rep2 sig c d } \\
& \rightarrow \text { Rep2 sig }(\text { WTree a c) (WTree b d) }
\end{aligned}
$$

Figure 6. Type representation for generic functions of arity two

$$
\text { data } \operatorname{Rep} 2(\operatorname{sig}:: \star \rightarrow \star \rightarrow \star)(\mathrm{a}:: \star)(\mathrm{b}:: \star)=\ldots
$$

and a new definition:

$$
\begin{aligned}
& \text { gmap :: Rep2 Map a } \mathrm{b} \rightarrow \mathrm{a} \rightarrow \mathrm{b} \\
& \text { gmap }=\ldots
\end{aligned}
$$

Now, what happens if we want to define generic zip? The signature abstracts over three variables: newtype Zip a b c $=Z i p(a \rightarrow$ $\mathrm{b} \rightarrow \mathrm{c})$, which needs a new representation:

$$
\text { data Rep3 }(\operatorname{sig}:: \star \rightarrow \star \rightarrow \star \rightarrow \star)(a:: \star)(b:: \star)(c:: \star)=\ldots
$$

The number of variables over which a generic function signature abstracts is called the arity of the function. Generic equality has arity one, while generic map has arity two. Unfortunately, arities are a source of redundancy: every arity requires a new representation. It is possible to define generic functions of arity one and two using Rep3, but that solution is rather inelegant.

Let us examine the increased generality of these new representations. The definition of Rep2, which is given in Figure 6, reveals strong similarities to Rep: there are representations for units, sums and products. The novelty lies in the Var2 constructor. This constructor stores a function having type sig a b. This is the key that enables abstraction over type constructors of arbitrary kind. To see why, let us try to specialize gmap to WTree, which has kind $\star \rightarrow \star \rightarrow \star$.

$$
\begin{aligned}
& \text { mapWTree }::(\mathrm{a} \rightarrow \mathrm{b}) \rightarrow(\mathrm{w} \rightarrow \mathrm{t}) \rightarrow \text { WTree a } \mathrm{w} \rightarrow \text { WTree } \mathrm{b} \mathrm{t} \\
& \text { mapWTree } f \mathrm{~g}= \text { let } m k R x=R \operatorname{Var} 2(\text { Map } x) \\
& \text { in } \operatorname{gmap}(r W \text { Tree2 }(\operatorname{mkR} f)(\text { mkR } g))
\end{aligned}
$$

The functional arguments which perform the transformation on the tree data are stored inside the representation, so that gmap can apply them when handling the RVar2 case.

New representations for differently-kinded types are not required, because the instantiation looks very similar to what we saw above in mapWTree. Hence it is more general than kind-specific representations like $\operatorname{Rep}^{\prime}$.

\section{Criteria}

In this section we describe the criteria used to evaluate the generic programming libraries. We have grouped criteria around three aspects:

- Types: To which datatypes generic functions can be applied, and the signatures of generic functions.

- Expressiveness: The kind of generic programs that can be written.

- Usability: How convenient a library is to use, efficiency, quality of library distribution, portability. 


$\begin{array}{lcl}\text { Types } & \text { Expressiveness (continued) } & \text { Usability } \\ \text { - Universe Size } & \text { - Ad-hoc definitions for } & \text { - Performance } \\ \text { - Subuniverses } & \text { datatypes } & \text { - Portability } \\ \text { Expressiveness } & \text { - Ad-hoc definitions for } & \text { - Overhead of } \\ \text { - First-class } & \text { - Exstructors } & \text { library use } \\ \text { generic } & \text { - Multiple arguments } & \text { - Practical aspects } \\ \text { functions } & \text { - Eultiple type of use and } \\ \text { - Abstraction } & \text { representation arguments } & \text { learning } \\ \begin{array}{l}\text { over type } \\ \text { constructors }\end{array} & \text { - Constructor names } & \\ \text { - Separate } & \text { - Consumers, transformers, } & \\ \text { compilation } & \text { and producers } & \end{array}$

Figure 7. Criteria overview

Figure 7 summarizes the criteria and the organization. In this section, we describe the evaluation criteria and, when possible, we illustrate them with code.

\section{Types}

- Universe Size. What are the types that a generic function can be used on? The more types a generic function can be used on, the bigger the universe size for that library. Different approaches implement generic universe extension in different ways, hence the sizes of their universes can differ.

Ideally, we would like to know whether a given library supports generic extension to nested and higher-kinded datatypes. But the claim that universe extension applies to, for example, nested datatypes is impractical to verify. It would require a rigorous proof that covers all nested datatypes.

Instead, we take a less ambitious alternative to estimate the size of the universe. We test whether a given approach supports extension to a number of datatypes, each of which demonstrates a particular datatype property. We test universe extension on lists and BinTree (regular datatypes), GRose (higherkinded), Perfect (nested), NGRose (higher-kinded and nested), and Company datatypes and WTree (other Haskell 98, that is, none of the previous categories).

- Subuniverses. Is it possible to restrict the use of a generic function on a particular set of datatypes, or on a subset of all datatypes? Will the compiler flag uses on datatypes outside that subuniverse as a type error?

\section{Expressiveness}

- First-class generic functions. Can a generic function take a generic function as an argument? This is tested by gmap $Q$, the function that applies a generic function argument to all constructor arguments:

$$
\begin{aligned}
& \text { gmapQ (rList RInt) gshow }(1:[2]) \\
& \quad \rightsquigarrow[\text { gshow RInt } 1, \text { gshow (rList RInt })[2]] \\
& \rightsquigarrow[" 1 ", "(:) 2 \text { []"] }
\end{aligned}
$$

Here gshow is applied to the two fields of the list constructor $(:)$, each having a different type, hence gshow must be instantiated to different types.

- Abstraction over type constructors. The equality function can usually be defined in an approach to generic programming, but a generalisation of the map function on lists to arbitrary container types cannot be defined in all proposals. This criterion is tested by the gmap and crushRight generic functions.

- Separate compilation. Is generic universe extension modular? That is, can a datatype defined in one module be used with a generic function and type representation defined in other modules without the need to modify or recompile them? This criterion is tested by applying generic equality to BinTree, which is defined in a different module than equality and the library itself.

$$
\begin{aligned}
& \text { module BinTreeE } q \text { where } \\
& \begin{array}{l}
\text { import } L I G D \quad-\text { import LIGD representations } \\
\text { import } G E q \quad-- \text { and geq } \\
\text { data BinTree a }=\ldots \\
\text { rBinTree } r_{\mathrm{a}}=\text { RType }(\ldots) \\
\quad(\text { EP fromBinTree toBinTree }) \\
\text { eqBinTree }=g e q \\
(\text { rBinTree RInt }) \\
(\text { Leaf } 2) \\
(\text { Bin }(\text { Leaf } 1)(\text { Leaf } 3))
\end{array}
\end{aligned}
$$

- Ad-hoc definitions for datatypes. Can a generic function contain specific behaviour for a particular datatype, and let the remaining datatypes be handled generically? In this situation, adhoc, datatype-specific definitions are used instead of uniformly generic behaviour. This is tested by the selectSalary function, which consists of cases that perform a traversal over a datatype, accumulating the values collected by the Salary ad-hoc case (traversal code omitted for brevity):

$$
\begin{aligned}
& \text { selectSalary }:: \text { Rep a } \rightarrow \text { a } \rightarrow[\text { Salary }] \\
& \text { selectSalary } R \text { Salary }(S x)=[S x]
\end{aligned}
$$$$
\cdots
$$

- Ad-hoc definitions for constructors. Can we give an ad-hoc definition for a particular constructor, and let the remaining constructors be handled generically? This is tested by the rmWeights function, which should have an explicit case to remove WithWeight constructors and the remaining constructors should be handled generically.

- Extensibility. Can the programmer non-generically extend the universe of a generic function in a different module? Because the extension meant here is non-generic, this criterion makes sense only if ad-hoc cases are possible. This criterion is tested by extending gshow with an ad-hoc case that prints lists using Haskell notation:

$$
\begin{aligned}
& \text { module ExtendedGShow where } \\
& \text { import GShow -- import definition of gshow } \\
& \text {-- ad-hoc extension } \\
& \text { geq (RList } \left.r_{\mathrm{a}}\right) \text { xs }=\ldots
\end{aligned}
$$

- Multiple arguments. Consumer functions such as gshow and selectSalary have one argument that is generic. Can the approach define a function that consumes more than one generic argument, such as the generic equality function?

- Multiple type representation arguments. Can a function be generic in more than one type? That is, can a generic function, such as the generic transpose function, receive two or more type representations?

The evaluation of this criterion is work in progress, so we do not include it in this paper.

- Constructor names. Can the approach provide the names of the constructors to which a generic function is applied? This is tested by the gshow generic function.

- Consumers, transformers, and producers. Is the approach capable of defining generic functions that are:

- consumers $(\mathrm{a} \rightarrow \mathrm{T})$ : gshow and selectSalary 
- transformers ( $\rightarrow$ a or $\mathrm{a} \rightarrow \mathrm{b}$ ): updateSalary and gmap

- producers $(\mathrm{T} \rightarrow \mathrm{a})$ : gfulltree

\section{Usability}

- Performance. Some proposals use many higher-order functions to implement generic functions, others use conversions between datatypes and structure types. We have compared running times for some of the test functions for the different libraries.

- Portability. Few proposals use only the Haskell98 standard for implementing generic functions, most use all kinds of (sometimes unimplemented) extensions to Haskell98, such as recursive type synonyms, multi-parameter type classes with functional dependencies, GADTs, etc. A proposal that uses few or no extensions is easier to port across different Haskell compilers.

- Overhead of library use. How much overhead (additional programming effort) is imposed on the programmer for the use of a generic programming library? We are interested in (1) support for automatic generation of structure representations, (2) number of structure representations needed per datatype, (3) the amount of work to instantiate a generic function, and (4) the amount of work to define a generic function.

- Practical aspects. Does there exist an implementation? Is it maintained? Is it documented?

- Ease of learning and use. Some generic programming libraries use implementation mechanisms that make their use or learning more difficult.

\subsection{Coverage of testable criteria}

Criteria can be divided into testable and non-testable groups. Testable criteria are the ones that can be tested by means of a generic function in the benchmark suite. Figure 8 shows the coverage of testable criteria. The rows represent testable criteria and the columns represent the means of testing them. The first group of columns stand for the testing functions introduced in Section 3. The criteria that a generic function tests are marked with $\bullet$.

The second group of columns stand for datatypes that test generic universe extension. These tests check whether generic equality can be instantiated and applied to values of those types. This explains that these columns test for the same criteria that the geq column does.

Some testing functions unavoidably require support of two criteria from a library. For example, the generic extension test on the GRose datatype requires separate compilation and higher-kinded datatypes. This brings up the problem that lack of support for the first criterion will cause failure of the test, which, according to our procedure (described in Section 3), means failure for the second criterion too. As a result, despite the fact that the second criterion remains untested, the criterion will be assumed non-supported by the library. This test would fail on Spine because it does not support separate compilation, but from the failure of the test it can erroneously be concluded that higher-kinded datatypes are not supported by Spine.

For this reason we have avoided requiring more than one criterion to implement a testing function, but this is not always possible. In such a situation we cheat a little: we ignore the issue of separate compilation and test it separately. This is shown in Figure 8. The criteria that are normally needed but are ignored for the particular test (because of the more than one criterion per test issue) are marked with $\bigcirc$.

\subsection{Design choices}

The criteria that we have seen so far are interesting from a user's point of view. They inform the user on what generic programs can and cannot be written using the libraries. However, it is also illustrative to see the design choices that have been taken by the designers of these libraries, because a particular design choice may improve or hinder the support of an expressiveness criterion. For example, the use of type classes is essential to libraries that support extensibility, but they can also make the use of generic functions as first-class values more difficult.

In this paper, we look at two design choices:

- Implementation mechanisms. How are types and their structure represented at runtime? Are these representations handled explicitly (as arguments that can be pattern matched) or implicitly (as type class contexts)? Are they abstract (higher order, including functions) or concrete (first order syntax for types).

- Views. What are the views that the generic library supports? Examples of views are the sum of products view, the fixed point view, and the spine view. A library typically includes a type representation per view.

A third possible design choice is whether generic functions are instantiated by compile time specialisation or by interpretation of type representations at runtime. Here we do not include this design choice, because all evaluated libraries use interpretation. Approaches that encode type and structure representations as datatypes are clearly doing interpretation of the representation values. Type class based approaches also perform interpretation: dictionary values are used at runtime. Of course, some specialisation may take place if the compiler performs inlining in the generic program.

Why is this design decision important? If an approach would implement instantiation by compile-time specialisation, that approach would most likely not support higher-order generic functions. This is because higher-orderness requires specialising the generic function argument at runtime, as opposed to compiled time. Yet, it is interesting to see that some type class based libraries, namely EMGM, have difficulties supporting higher order functions.

\section{Evaluation summary}

We have tried to implement the benchmark in each of the generic programming libraries. This section gives a summary of the results. Figure 9 presents the results in a table. The criteria that a generic programming library supports are marked with $\boldsymbol{\bullet}$. The ones that are not supported are marked with $\bigcirc$. If a criterion is partially supported, or if it requires unusual programming effort, it is marked with $\boldsymbol{O}$. A more detailed evaluation and a discussion on the design choices can be found in Section 5. That section also discusses the design choices behind each of the evaluated libraries.

Universe Size. The PolyLib library is limited to regular datatypes (with one parameter). In RepLib, the datatypes with higher-kinded arguments (GRose and NGRose) are not supported. Approaches such as SYB, SYB3, Uniplate, and EMGM, which are based on type classes, have trouble supporting NGRose; the three first do not support it at all, while EMGM supports it but loses some functionality. Furthermore, the SYB3 library does not support Perfect and it has an additional complication: BinTree is supported only if the instance is manually written, but not with the generated instance; we return to this problem when evaluating the generation of representations. LIGD and Spine have the advantage of a large universe size: they support all datatypes in this test. Smash also supports all datatypes, but there are datatypes that require unusual effort to allow generic extension: Perfect, NGRose, and Company. 


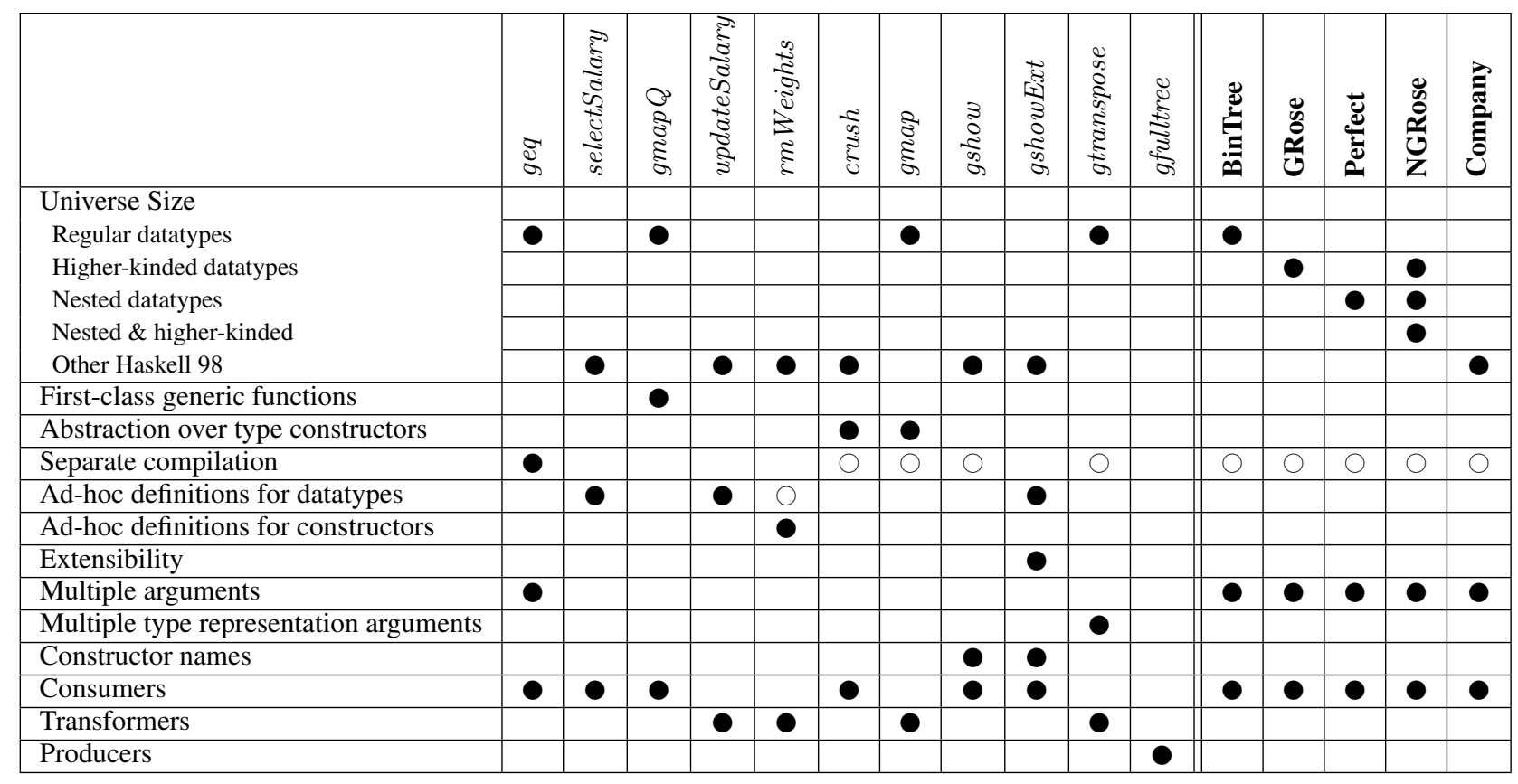

- The criterion is tested by the example: the criterion is needed to implement test.

$\bigcirc$ The criterion is normally needed by test, but it is circumvented to test other criteria.

Figure 8. Functions and datatypes set out against criteria.

Subuniverses. The PolyLib, EMGM, and RepLib libraries support subuniverses.

First-class generic functions. In LIGD, SYB, and Spine a generic function is a polymorphic Haskell function, so they are first-class values. The PolyLib and Uniplate libraries do not support this criterion. The SYB3, EMGM, and RepLib libraries support this criterion, but in the second there is additional complexity so it only scores sufficient. Smash requires a new structure representation for this test so it only scores sufficient.

Abstraction over type constructors. The LIGD, PolyLib, EMGM, RepLib, Spine, and Smash libraries support abstraction over type constructors. However, PolyLib, and Spine only support abstraction over type constructors of kind $\star \rightarrow \star$, so the support of these approaches for this criterion is only sufficient. The SYB, SYB3, and Uniplate libraries do not support this criterion.

Separate compilation. The libraries that support separate compilation are LIGD, PolyLib, SYB, SYB3, EMGM, RepLib, Smash, and Uniplate. The only evaluated approach that does not support this criterion is Spine: generic universe extension requires recompilation.

Ad-hoc definitions for datatypes. This criterion is supported by SYB, SYB3, EMGM, RepLib, Smash, and Uniplate, but not by LIGD and Spine. PolyLib supports ad-hoc cases for regular datatypes, but it cannot be applied to Company values, so it fails the test.

Ad-hoc definitions for constructors. Ad-hoc definitions for constructors are supported by LIGD, SYB, SYB3, Spine, EMGM, RepLib, Smash, and Uniplate. However, in LIGD the structure representation has to be adapted for this criterion to work. Because of this additional complication this approach scores sufficient.
Extensibility. This criterion is supported by SYB3, EMGM, and RepLib. It is only partially supported by PolyLib, because it works only for regular datatypes, and hence it fails for this test.

Multiple arguments. Multiple argument functions are supported by almost all approaches, however, in some approaches, such as SYB and SYB3, the definitions can be rather complex, and therefore they score sufficient. In Smash the definition is not complex, but it requires a separate structure representation. The only library that fails to support equality is Uniplate.

Constructor names. Constructor names are supported by all evaluated approaches except Uniplate.

Consumers, transformers, and producers. Almost all libraries support definitions of functions in the three categories. However, there are libraries that use different structure representations for consumers and producers such as SYB, SYB3, Spine, and Smash. Smash in addition uses a different structure representation for transformations. Uniplate does not support producer functions.

Performance. We have used some of the test functions for a performance benchmark comparing running times for larger inputs. The results are very sensitive to small code differences and compiler optimizations so firm conclusions are difficult to draw, but the best overall performance score is shared between EMGM, Smash, and Uniplate.

Portability. The three most portable approaches are LIGD, EMGM, and Uniplate. The first approach relies on existential types and the other two on multi-parameter type classes, both extensions are very likely to be included in the next Haskell standard. Furthermore, multi-parameter type classes in EMGM are used in a non-essential way: the functionality of EMGM would only be slightly affected in their absence. The other approaches rely on non-portable Haskell extensions. 


\begin{tabular}{|c|c|c|c|c|c|c|c|c|c|}
\hline & LIGD & PolyLib & SYB & SYB3 & Spine & EMGM & RepLib & Smash & Uniplate \\
\hline $\begin{array}{l}\text { Universe Size } \\
\text { Regular datatypes } \\
\text { Higher-kinded datatypes } \\
\text { Nested datatypes } \\
\text { Nested \& higher-kinded } \\
\text { Other Haskell } 98 \\
\text { Subuniverses }\end{array}$ & $\begin{array}{l}\bullet \\
\bullet \\
\bullet \\
0 \\
0 \\
0\end{array}$ & $\begin{array}{l}0 \\
0 \\
0 \\
0 \\
0 \\
0\end{array}$ & $\begin{array}{l}\bullet \\
\bullet \\
0 \\
0 \\
0 \\
0\end{array}$ & $\begin{array}{l}0 \\
0 \\
0 \\
0 \\
0 \\
0\end{array}$ & $\begin{array}{l}\bullet \\
\bullet \\
\bullet \\
0 \\
0\end{array}$ & $\begin{array}{l}0 \\
0 \\
0 \\
0 \\
0 \\
0\end{array}$ & $\begin{array}{l}\bullet \\
0 \\
\bullet \\
0 \\
\bullet \\
0\end{array}$ & $\begin{array}{l}0 \\
0 \\
0 \\
0 \\
0 \\
0\end{array}$ & $\begin{array}{l}0 \\
0 \\
0 \\
0 \\
0 \\
0\end{array}$ \\
\hline $\begin{array}{l}\text { First-class generic functions } \\
\text { Abstraction over type constructors } \\
\text { Separate compilation } \\
\text { Ad-hoc definitions for datatypes } \\
\text { Ad-hoc definitions for constructors } \\
\text { Extensibility } \\
\text { Multiple arguments } \\
\text { Constructor names } \\
\text { Consumers } \\
\text { Transformers } \\
\text { Producers }\end{array}$ & $\begin{array}{l}0 \\
\bullet \\
\bullet \\
0 \\
0 \\
0 \\
0 \\
0 \\
0 \\
0 \\
0\end{array}$ & $\begin{array}{l}0 \\
0 \\
0 \\
0 \\
0 \\
0 \\
0 \\
0 \\
0 \\
0 \\
0\end{array}$ & $\begin{array}{l}0 \\
0 \\
\bullet \\
\bullet \\
0 \\
0 \\
0 \\
0 \\
0 \\
0 \\
0\end{array}$ & $\begin{array}{l}\bullet \\
0 \\
\bullet \\
\bullet \\
\bullet \\
0 \\
0 \\
0 \\
0 \\
0 \\
0\end{array}$ & $\begin{array}{l}0 \\
0 \\
0 \\
0 \\
0 \\
0 \\
\bullet \\
0 \\
0 \\
0 \\
0\end{array}$ & $\begin{array}{l}1 \\
\bullet \\
\bullet \\
: \\
: \\
: \\
: \\
: \\
\bullet \\
\bullet\end{array}$ & $\begin{array}{l}0 \\
\bullet \\
: \\
: \\
: \\
: \\
: \\
: \\
0 \\
0\end{array}$ & $\begin{array}{l}0 \\
0 \\
0 \\
0 \\
0 \\
0 \\
0 \\
0 \\
0 \\
0 \\
0\end{array}$ & $\begin{array}{l}0 \\
0 \\
\bullet \\
\bullet \\
\bullet \\
0 \\
0 \\
0 \\
0 \\
0 \\
0\end{array}$ \\
\hline $\begin{array}{l}\text { Performance } \\
\text { Portability } \\
\text { Overhead of library use }\end{array}$ & D & $\begin{array}{l}\mathbf{D} \\
0\end{array}$ & $\begin{array}{l}0 \\
0\end{array}$ & $\begin{array}{l}0 \\
0\end{array}$ & $\begin{array}{l}0 \\
0\end{array}$ & $\bullet$ & & $\begin{array}{l}0 \\
0\end{array}$ & • \\
\hline $\begin{array}{l}\text { Automatic generation of representations } \\
\text { Number of structure representations } \\
\text { Work to instantiate a generic function } \\
\text { Work to define a generic function } \\
\text { Practical aspects } \\
\text { Ease of learning and use }\end{array}$ & $\begin{array}{l}0 \\
4 \\
0 \\
0 \\
0 \\
0\end{array}$ & $\begin{array}{l}0 \\
1 \\
\bullet \\
0 \\
0 \\
0\end{array}$ & $\begin{array}{l}\bullet \\
2 \\
\bullet \\
\bullet \\
\bullet \\
0\end{array}$ & $\begin{array}{l}0 \\
2 \\
0 \\
0 \\
0 \\
0\end{array}$ & $\begin{array}{l}0 \\
3 \\
0 \\
0 \\
0 \\
0\end{array}$ & $\begin{array}{l}0 \\
4 \\
0 \\
0 \\
0 \\
0\end{array}$ & $\begin{array}{l}\mathbf{0} \\
4 \\
0 \\
0 \\
0 \\
0\end{array}$ & $\begin{array}{l}0 \\
8 \\
0 \\
0 \\
0 \\
0\end{array}$ & $\begin{array}{l}\bullet \\
1 \\
\bullet \\
\bullet \\
\bullet \\
\bullet\end{array}$ \\
\hline
\end{tabular}

Figure 9. Evaluation of generic programming approaches

Overhead of library use. The SYB, SYB3, RepLib, and Uniplate libraries are equipped with automatic generation of representations. However, automatic generation in RepLib fails for type synonyms. In SYB3, the generated Data instance for BinTree causes nontermination when used with generic equality.

The number of structure representations is high for libraries such as LIGD, EMGM, and RepLib. The reason is that type constructor abstraction in these approaches requires one representation per generic function arity. The number of representations in Smash is even higher due to the amount of relatively specialized representations. More information can be found in Section 5. The SYB, SYB3, and Spine approaches have one representation for consumers and another for producers. In addition, Spine has a representation to abstract over type constructors. PolyLib and Uniplate have only one representation.

The instantiation of a generic function is easier (for the programmer) in libraries that support implicit type representations, such as PolyLib, SYB, SYB3, Uniplate, Smash, EMGM, and RepLib. However, the last two libraries require additional effort to enable instantiation. Therefore PolyLib, SYB, SYB3, Smash, and Uniplate are the libraries that require the least effort to instantiate a generic function.

The work required to define a generic function is higher, in the sense that more implementation machinery is required, in LIGD, SYB3, and RepLib.

Practical aspects. The SYB, RepLib, and Uniplate libraries have well-maintained and documented distributions. PolyLib has an official distribution, but it is not maintained anymore. The SYB3 library has two distributions: one does not compile under some versions of GHC $(6.6,6.8 .1,6.8 .2)$ and the other does not have a number of useful combinators. Smash has an online distribution, but its interface is not as structured as, for example, SYB3. The remaining approaches, LIGD, Spine, and EMGM, do not have a well-maintained distribution.

Ease of learning and use. It is hard to determine how easy it is to learn how to use a library. We approximate this criterion by looking at the mechanisms used in the implementation of the libraries. We consider an approach easier if its implementation mechanisms are relatively simple such as for PolyLib and Uniplate (type classes), and Spine (GADTs). An approach is relatively difficult if it uses sophisticated implementation mechanisms, for example rank-2 typed combinators and abstraction over type classes as in SYB3. Intermediate approaches use advanced mechanisms only occasionally. One such approach is EMGM, which uses arity-based representations. More information can be found in Section 5.

\section{Evaluation}

A generic programming library provides an interface to achieve generic programming behaviour and uses certain mechanisms to implement it. These interfaces and mechanisms correspond to the concepts that we introduced in Section 2. In particular we can usually identify mechanisms corresponding to type representations, structure representations and functions that act on them. So two libraries may implement generic behaviour in very different ways, by providing different ways to encode structure representations, for example.

Before proceeding with the detailed evaluation we introduce below each of the compared libraries and relate them against the concepts introduced in Section 2. We focus in particular on how they implement case analysis on types and structure representation of datatypes. 


\subsection{Lightweight implementation of Generics and Dynamics}

The Lightweight implementation of Generics and Dynamics (LIGD) library was introduced by Cheney and Hinze [2002]. The presentation in the current paper largely follows the original presentation of LIGD. The difference is that the original Rep is not a GADT but a normal datatype. This datatype encodes the GADT by including conversion functions in the datatype constructors and by the use of existential types.

The generic view that LIGD uses is the sum of products view. The original LIGD paper does not include a view to abstract over type constructors, but it is well known how to do so: Hinze and Löh [2007] present a variant of LIGD called dictionary-passing style that abstracts over type constructors.

In this library case analysis on types is performed by means of pattern matching. The type structure of datatypes is represented by the RType constructor.

\subsection{PolyLib}

The pre-processor-based language extension PolyP [Jansson and Jeuring, 1997, 1998] was later packaged up as a more lightweight library [Norell and Jansson, 2004] and this library is what we compare in this paper. The library is limited to regular datatypes (with one parameter) so the supported universe is relatively small. But the smaller universe makes it possible to express a wider range of generic functions - the library contains definitions of folds and unfolds, traversals and even functions generic in two type parameters such as transpose $:: \ldots \Rightarrow d(e a) \rightarrow e(d a)$.

The limited universe means that PolyLib is not suitable as a general generic library - it is included here as a "classic reference" and because of its expressiveness.

PolyLib uses a combination of the fixed-point view and the sum of products view. Each TIF is defined as a type (constructor) class with one instance for each universe building block (unit, sum, product, composition, function, const, parameter, and recursion).

\subsection{Scrap your boilerplate}

In the Scrap your boilerplate (SYB) library [Lämmel and Peyton Jones, 2003, 2004] generic functions are not programmed by pattern matching on the structural representation of a value, but rather by means of combinators. There are combinators for doing case analysis on types and for inspecting the structure of values.

Case analysis combinators exist in several variants: query combinators, transformation combinators, and monadic tranformations combinators amongst others. Let us consider the combinators for queries: $m k Q$ and $\operatorname{ext} Q$.

$$
\begin{aligned}
m k Q & :: \text { (Typeable a, Typeable } \mathrm{b}) \\
& \Rightarrow \mathrm{r} \rightarrow(\mathrm{b} \rightarrow \mathrm{r}) \rightarrow \mathrm{a} \rightarrow \mathrm{r} \\
\operatorname{ext} Q & ::(\text { Typeable } \mathrm{a}, \text { Typeable } \mathrm{b}) \\
& \Rightarrow(\mathrm{a} \rightarrow \mathrm{r}) \rightarrow(\mathrm{b} \rightarrow \mathrm{r}) \rightarrow(\mathrm{a} \rightarrow \mathrm{r})
\end{aligned}
$$

The $m k Q$ combinator takes an ad-hoc case specific to $\mathrm{b}$ values and creates a polymorphic function that can be applied to any value a that is an instance of Typeable. If it is applied to $a b$ value -that is, if at runtime it is determined that $a$ and $b$ are the same type- the function with type $b \rightarrow r$ is applied to it, otherwise the argument of type $r$ is returned. The $\operatorname{ext} Q$ combinator extends a polymorphic query built with $m k Q$ with yet another ad-hoc case.

These combinators are implemented by means of type-safe casting, which ultimately relies on the function unsafeCoerce, an unsafe Haskell extension that converts a value from one type to any other type. They also rely on the Typeable type class, which provides runtime representations of types, so that the equality of two types can be established at runtime.

The structure of datatypes is represented by the higher-order combinators gfoldl and gunfoldl. These are used to write con- sumer and producer functions respectively. Datatypes whose structure is represented are instances of the Data type class.

$$
\begin{aligned}
\text { class Typeable } \mathrm{a} & \Rightarrow \text { Data a where } \\
\text { gfoldl :: Data } \mathrm{a} & \Rightarrow(\forall \mathrm{a} \mathrm{b} . \text { Data } \mathrm{b} \Rightarrow \mathrm{w}(\mathrm{a} \rightarrow \mathrm{b}) \\
& \rightarrow(\forall \mathrm{a} \cdot \mathrm{a} \rightarrow \mathrm{w} \mathrm{a}) \\
& \rightarrow \mathrm{w} \mathrm{b}) \\
& \rightarrow \mathrm{wa}
\end{aligned}
$$

The instance for lists below makes gfoldl clearer.

$$
\begin{aligned}
& \text { instance Data a } \Rightarrow \text { Data [a] where } \\
& \begin{aligned}
\text { gfoldl } k z[] & =z[] \\
\text { gfoldl } k z(x: x s) & =\left(z(:)^{\prime} k^{6} x\right)^{\prime} k^{\prime} x s
\end{aligned}
\end{aligned}
$$

The gfoldl function applies $z$ to the constructor and applies the result to the arguments of the constructor using $k$. In essence, gfoldl exposes the constructor and the arguments to the $k$ and $z$ functions. Further explanations can be found in the original SYB paper and in the description of the Spine approach below.

The SYB library provides two structure representations of data, one through gfoldl for consumer functions, and another through gunfoldl for producer functions. Because of their types these functions need support for rank-2 polymorphism.

As an example, this is how selectSalary is implemented in SYB:

$$
\begin{aligned}
& \text { selectSalary }:: \text { Data a } \Rightarrow \text { a } \rightarrow[\text { Salary }] \\
& \text { selectSalary } x=\left([]^{\prime} m k Q \text { ' salaryCase }\right) x: \\
& \text { concat }(\text { gmapQ selectSalary } x)) \\
& \text { where salaryCase }(\text { sal }:: \text { Salary })=[\text { sal }]
\end{aligned}
$$

The $m k Q$ expression performs case analysis on types, $x$ is added to the result if it is a Salary but not otherwise. Then $\operatorname{gmap} Q$ applies selectSalary to the children of $x$.

$$
\operatorname{gmap} Q:: \text { Data } \mathrm{a} \Rightarrow(\forall \mathrm{a} . \text { Data } \mathrm{a} \Rightarrow \mathrm{a} \rightarrow \mathrm{u}) \rightarrow \mathrm{a} \rightarrow[\mathrm{u}]
$$

\subsection{Scrap your boilerplate, extensible with typeclasses}

A serious drawback of Scrap your boilerplate is that it is not extensible: once a function (such as selectSalary) is defined, it cannot be extended with an ad-hoc case. This problem is solved by the extensible variant of Scrap your boilerplate (SYB3) [Lämmel and Peyton Jones, 2005]. The extended approach is still combinator based, indeed generic functions are written using combinators such as $g$ foldl and $g$ map $Q$.

$$
\begin{aligned}
& \operatorname{class}(\text { Typeable a, Sat }(\mathrm{ctx} \mathrm{a})) \Rightarrow \text { Data ctx a where } \\
& \text { gfoldl }:: \text { Proxy ctx } \\
& \rightarrow(\forall \mathrm{b} \mathrm{c} \cdot \text { Data ctx } \mathrm{b} \Rightarrow \mathrm{w}(\mathrm{b} \rightarrow \mathrm{c}) \rightarrow \mathrm{b} \rightarrow \mathrm{wc}) \\
& \rightarrow(\forall \mathrm{g} \cdot \mathrm{g} \rightarrow \mathrm{w} \mathrm{g}) \\
& \rightarrow \mathrm{a} \rightarrow \mathrm{w} \mathrm{a} \\
& \operatorname{gmap} Q:: \text { Proxy ctx } \\
& \rightarrow(\forall \mathrm{a} . \text { Data ctx } \mathrm{a} \Rightarrow \mathrm{a} \rightarrow \mathrm{r}) \rightarrow \mathrm{a} \rightarrow[\mathrm{r}]
\end{aligned}
$$

There is an additional type argument ctx to Data. This is the essential ingredient that allows extension. Both functions also take an additional Proxy argument, which is merely a way to inform the type checker what ctx type is used and the actual argument value is not important. We shall explain more about ctx shortly.

However, case analysis on types is no longer based on combinators such as $m k Q$ and $e x t Q$. Instead, generic functions are defined as a type class and ad-hoc cases are given as instances. Consider selectSalary, for example:

$$
\begin{aligned}
& \text { class SelectSalary a where } \\
& \text { selectSalary }:: \mathrm{a} \rightarrow[\text { Salary }]
\end{aligned}
$$


The generic function is the method of a type class. It follows that case analysis on types is performed by the type class system. We show below how to write the generic and the Salary-specific cases:

$$
\text { -- case for Salary }
$$

instance SelectSalary Salary where

selectSalary sal $=[$ sal $]$

-- generic case, not complete yet

instance Data SelectSalary a $\Rightarrow$ SelectSalary a where selectSalary $x=$ gmap $Q$ someProxy

$$
\text { (...) } x
$$

The idea of the generic case is that we want to apply selectSalary recursively to the top-level sub-trees in $x$. Since $g$ map $Q$ abstracts over the generic function that is applied, it follows that it has to abstract over the type class as well. However, Haskell does not support abstraction over type classes, so in this approach abstraction over type classes is emulated by means of dictionaries.

The first step is to define a dictionary datatype that represents SelectSalary instances.

$$
\begin{aligned}
& \text { data SelectSalaryD a } \\
& \quad=\text { SelectSalary } D\{\text { selectSalary } D:: \mathrm{a} \rightarrow[\text { Salary }]\}
\end{aligned}
$$

Next, every generic function definition must include a Sat instance declaration. The Sat type class is used to enable SYB3 combinators to construct dictionaries of generic functions.

$$
\begin{aligned}
& \text { class Sat a where }\{\text { dict }:: \text { a }\} \\
& \text { instance SelectSalary a } \Rightarrow \text { Sat (SelectSalaryD a) where } \\
& \quad \text { dict }=\text { SelectSalaryD selectSalary }
\end{aligned}
$$

Finally, combinators that take generic functions as arguments, such as $\operatorname{gmap} Q$, include Sat in their context to abstract over the dictionary argument. This becomes clearer in the definition of $g \operatorname{map} Q$ for lists:

$$
\begin{aligned}
& \text { instance }(\text { Sat }(\operatorname{ctx}[\mathrm{a}]), \text { Data ctx a }) \Rightarrow \text { Data ctx }[\mathrm{a}] \text { where } \\
& \operatorname{gmap} Q-f[]=[] \\
& g \operatorname{map} Q-f(x: x s)=\left[\begin{array}{lll}
f x, f x
\end{array}\right]
\end{aligned}
$$

The generic case of selectSalary looks as follows:

$$
\begin{aligned}
& \text { instance Data SelectSalaryD a } \Rightarrow \text { SelectSalary a where } \\
& \text { selectSalary } x=\text { concat }(\text { gmap } Q \text { selectSalaryProxy } \\
& \text { (selectSalaryD dict) } x) \\
& \text { selectSalaryProxy }:: \text { Proxy SelectSalaryD } \\
& \text { selectSalaryProxy }=\text { undefined }
\end{aligned}
$$

This approach uses the same structure representation as SYB. However case analysis on types is implemented using the type class system, and hence it no longer uses type safe casts. However, casts are still used in the library, for example in the definition of the generic equality function.

\subsection{Scrap your boilerplate, spine view variant}

The Scrap your Boilerplate variant introduced in Hinze et al. [2006] replaces the combinator based approach of SYB by a tangible representation of the structure of values, which is embodied by the Spine datatype:

$$
\begin{aligned}
& \text { data Spine }: \star \star \rightarrow \star \text { where } \\
& \quad \text { Con }:: \mathrm{a} \rightarrow \text { Spine } \mathrm{a} \\
& \quad(\$):: \text { Spine }(\mathrm{a} \rightarrow \mathrm{b}) \rightarrow \text { Typed } \mathrm{a} \rightarrow \text { Spine } \mathrm{b}
\end{aligned}
$$

where the Typed representation is given by:

$$
\begin{aligned}
& \text { data Typed } \mathrm{a}=(:>)\{\text { typeOf }:: \text { Type a, val }:: \mathrm{a}\} \\
& \text { data Type }:: \star \rightarrow \star \text { where }
\end{aligned}
$$

$$
\begin{aligned}
& \text { IntR :: Type Int } \\
& \text { ListR :: Type a } \rightarrow \text { Type [a] } \\
& \quad \ldots
\end{aligned}
$$

This approach represents the structure of datatype values by making the application of a constructor to its arguments explicit. For example, the list $[1,2]$ can be represented by Con $(:): \$($ IntR $:>$ 1) :\$ (ListR IntR :> [2]).

Unlike in LIGD, there is no general purpose constructor like RType to support generic universe extension. Generic universe extension is achieved as follows: (1) the datatype must have a Type constructor that represents it, e.g. the ListR constructor for lists, and (2) the function toSpine that transforms a value to its structure representation must be extended to cover that type.

$$
\begin{aligned}
& \text { toSpine }:: \text { Type a } \rightarrow \text { a } \rightarrow \text { Spine a } \\
& \text { toSpine }(\text { ListR } t)[]=\text { Con }[] \\
& \text { toSpine }(\text { ListR } t)(x: x s)=\text { Con }(:): \$(t:>x) \\
& : \$(\text { ListR } t:>x s)
\end{aligned}
$$

$\cdots$

In Spine, case analysis on types is done as in LIGD, by pattern matching on Type values.

Generic and non-generic universe extension in Spine require recompilation of type representations and generic functions. For this reason Spine cannot be used as a library, and so it is a design pattern rather than a library. The authors of Spine also describe an extensible variant of Spine that is based on type classes (and therefore can be used as a library), but we do not evaluate it in this paper. This variant uses techniques similar to those in SYB3, so we expect that both libraries have similar expressiveness.

Producer generic functions cannot be defined using Spine. To solve this deficiency the authors introduced a "type spine view" in Hinze and Löh [2006]. In the evaluation we refer to both approaches as Spine. Both views, spine and the type spine view, correspond to gfoldl and gunfoldl in SYB. As the authors of Spine note, gfoldl is a fold over Spine values.

\subsection{Extensible and modular Generics for the masses}

The EMGM library [Hinze, 2006, Oliveira et al., 2006] does not use a datatype like Rep to represent types. Instead the type representations are encoded in the type class Generic, where every represented type has a corresponding method:

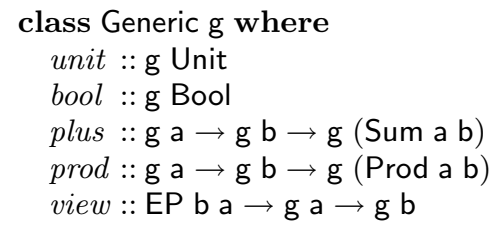

The type class abstracts over the signature of a generic function, here represented by $g$. To define a generic function, the programmer defines a type for the signature and then the definition is given in the instance declaration for that type. Consider, for example, the equality function. The signature type is defined as follows:

newtype Geq a $=\operatorname{Geq}\{g e q:: \mathrm{a} \rightarrow \mathrm{a} \rightarrow$ Bool $\}$

The definition of the generic function resides in the Geq instance declaration.

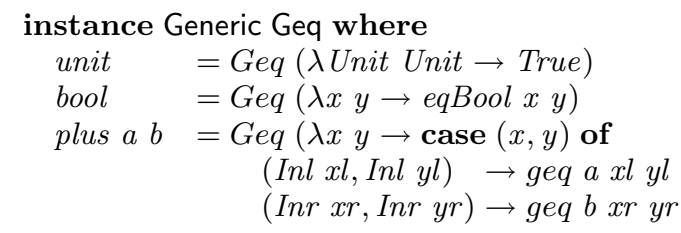




$$
\begin{aligned}
& \rightarrow \text { False } \\
& \operatorname{prod} a b=\overline{G e q}\left(\lambda\left(\operatorname{Prod} a_{1} b_{1}\right)\left(\operatorname{Prod} a_{2} b_{2}\right) \rightarrow\right. \\
& \text { geq } \left.a a_{1} a_{2} \wedge \text { geq } b b_{1} b_{2}\right) \\
& \text { view ep } a=\operatorname{Geq}(\lambda x y \rightarrow \text { geq a (from ep } x) \text { (from ep } y) \text { ) }
\end{aligned}
$$

The equality function is now defined for the universe of types comprising units, sums, products, and datatypes that have their structure represented by view, which is similar to the use of the RType constructor in LIGD. It follows that in EMGM case analysis on types is encoded using the methods of Generic. This is how the structure of lists is represented in EMGM.

$$
\begin{aligned}
& r \text { List }:: \text { Generic } \mathrm{g} \Rightarrow \mathrm{g} \text { a } \rightarrow \mathrm{g}[\mathrm{a}] \\
& \text { rList } a=\text { view listEP (unit'plus' (a'prod'rList } a))
\end{aligned}
$$

Extensibility of generic functions is achieved by means of defining sub-classes of Generic. For example, we define GenericList to enable ad-hoc definitions for lists:

$$
\begin{aligned}
& \text { class Generic } \mathrm{g} \Rightarrow \text { GenericList } \mathrm{g} \text { where } \\
& \text { list }:: \mathrm{g} \mathrm{a} \rightarrow \mathrm{g}[\mathrm{a}] \\
& \text { list }=r \text { List }
\end{aligned}
$$

The default implementation of GenericList uses the structure representation for lists. Therefore we can request generic behaviour for list equality with an empty instance declaration:

\section{instance GenericList Geq}

But we can also give a definition of equality specific to lists:

$$
\begin{gathered}
\text { instance GenericList Geq where } \\
\text { list geqa }=\operatorname{Geq}(\lambda x y \rightarrow \ldots)
\end{gathered}
$$

Now let us see how to apply generic equality:

$$
\text { geq (list bool) [ True, False] [True, True] } \rightsquigarrow \text { False }
$$

It is possible to make the use of generic functions easier by making the type representations implicit. This is achieved by means of a type class:

$$
\begin{aligned}
& \text { class GRep g a where } \\
& \text { over }:: \mathrm{g} \text { a } \\
& \text { instance Generic g } \Rightarrow \text { GRep g Unit where } \\
& \text { over }=\text { unit } \\
& \text { instance (Generic g, GRep g a, GRep g b) } \\
& \quad \Rightarrow \text { GRep g (Prod a b) where } \\
& \text { over }=\text { prod over over } \\
& \text { instance (GenericList g, GRep g a) } \Rightarrow \text { GRep g [a] where } \\
& \text { over }=\text { list over }
\end{aligned}
$$

Now, generic equality can be defined as follows:

$$
\text { gequal :: GRep Geq a } \Rightarrow \mathrm{a} \rightarrow \mathrm{a} \rightarrow \text { Bool }
$$$$
\text { gequal } x \text { y }=\text { geq over }
$$

\subsection{RepLib}

The RepLib library [Weirich, 2006] uses an ingenious combination of GADTs and type classes to implement generic functions. A generic function in this approach is implemented as a type class. Ad-hoc cases are given as an instance of this class. We use the gsum function from the original paper as an example.

$$
\begin{aligned}
& \text { instance GSum IntSet where } \\
& \text { gsum }(\operatorname{IntSet} x s)=\text { gsum }(\text { nub } x s)
\end{aligned}
$$

Here we give an ad-hoc case for sets of integers. This case eliminates duplicate elements and calls generic sum on the resulting list.

What makes gsum a generic function, and not-merely typeindexed, is the default implementation, which exploits the structure of datatypes:

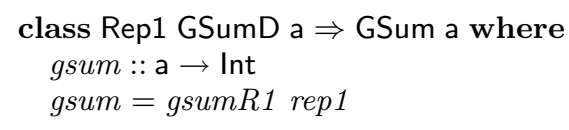

The structure representation for a is generated by rep 1 , a method of the Rep 1 type class.

$$
\begin{aligned}
& \text { class } \operatorname{Rep~a~} \Rightarrow \operatorname{Rep} 1 \text { c a where } \\
& \quad \text { rep } 1:: \operatorname{R} 1 \text { c } a
\end{aligned}
$$

Now, gsumR 1 can use the representation produced by rep 1 to process its argument of type a.

But what happens if gsumR 1 needs to recursively apply gsum to a substructure inside a? In RepLib such recursive calls are allowed by parametrising Rep1 over a dictionary type. In our example the dictionary is GSumD, which is defined as follows:

$$
\text { data GSumD a }=G \operatorname{Sum} D\{\text { gsumD }:: \mathrm{a} \rightarrow \text { Int }\}
$$

The representation produced by rep 1 contains GSumD dictionaries. These dictionaries package sum instances for a values. These instances are used when sum is applied recursively to the argument of a constructor, for example. To produce such dictionaries, the programmer defining the generic function is required to define a Sat instance for GSumD, it suffices to say that Sat is used in Rep1 instances to produce dictionaries. We give the instance definition for GSumD below:

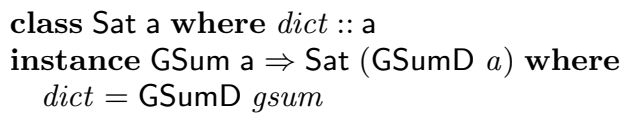

Note that this instance uses GSum to produce dictionaries. This means that even GSum instances defined in other modules are used. This is what allows RepLib generic functions to be extensible. The technique of explicit dictionaries to abstract over type classes is the same as in "Scrap your boilerplate with class".

The gsumR1 function is the structure-based definition of generic sum.

$$
\begin{aligned}
& \text { gsumR1 :: R1 GSumD a } \rightarrow \mathrm{a} \rightarrow \text { Int } \\
& \text { gsumR1 Int1 } \quad x=\ldots \\
& \text { gsumR1 (Arrow } \left.r_{1} r_{2}\right) \quad f=\ldots \\
& \text { gsumR1 (Data1 dt cons) } x \\
& =\text { case }(\text { findCon cons } x) \text { of } \\
& \quad \text { Val emb rec kids } \\
& \quad \rightarrow \text { foldl_l }(\lambda c a \text { a } b \rightarrow(\text { gsumD ca b) }+ \text { a) } 0 \text { rec kids } \\
& \text { gsumR1 } \quad x=0
\end{aligned}
$$

The two first cases and the last one correspond to integers, functions and all other cases respectively. Without going into detail, the third case uses the structure representation of the datatype (stored in Data1) to (1) find the representation for the constructor at hand (using findCon), (2) convert the constructor arguments into a heterogenous list, and (3) fold over this list applying generic sum to the constructor arguments (using fold $l_{-} l$ ). Note that the last step involves a recursive application of the function. Remember that the representation stores GSumD dictionaries for the constructor arguments. Suppose that $b$ (a constructor argument) has type $c$ and $c a$ has type GSumD c, then we can apply the dictionary function using gsumD.

In this approach the structure of datatypes is represented by GADTs such as R1. Case analysis over types is performed by the type class system, because generic functions are implemented as type classes (for example, GSum).

The RepLib library has an alternative way to implement case analysis on types. It provides SYB-style combinators such as $m k Q$ and $\operatorname{ext} Q$, these are implemented using type safe casts like in SYB. 
But these combinators use RepLib representations, rather than the Typeable class.

\subsection{Smash your boilerplate}

The 'Smash' approach is conceptually closely related to SYB. The latter uses a 'typecase' operation based on the run-time type representation (Typeable). The Smash approach uses a compiletime typecase operation. In both approaches, the structure of a new datatype is presented to the library (added to the universe) by declaring an instance of a special class: Data in SYB, LDat in Smash. A generic function is made of two parts. First, there is a term traversal strategy, identified by a label. One strategy may be to 'reduce' a term using a supplied reducing function (cf. fold over a tree). Another strategy may rebuild a term. The second component of a generic function is spec, the list of 'exceptions', or ad-hoc redefinitions. Each component of spec is a function that tells how to transform a term of a specific type. Exceptions override the generic traversal.

As an example, consider how selectSalary is defined in Smash:

$$
\begin{aligned}
& \text { selectSalary }:: \text { Company } \rightarrow \text { [Salary }] \\
& \text { selectSalary } x= \\
& \quad \text { gapp }\left(T L \_r e d \text { concat }\right)(\text { salaryCase }:+: \text { HNil) } x \\
& \text { where } \\
& \quad \text { salaryCase }:: \text { Salary } \rightarrow \text { [Salary }] \\
& \quad \text { salaryCase } s=[s]
\end{aligned}
$$

Here the library function gapp is applied to TL_red concat, which selects a bottom-up traversal (parametrised with concat) on $x$. This traversal applies one of the ad-hoc cases (second argument of gapp) to the nodes of $x$ being traversed. When traversing a node, the results of traversing the children are merged using the concat function. Note that ad-hoc cases are encoded as a heterogeneous list of functions. In the above example the list contains only one element.

This library implements case analysis using extensible record operations [Kiselyov et al., 2004], due to the way that ad-hoc cases are encoded. The structure representation is given once per datatype and per traversal strategy. To implement the functions in the test suite the following strategies are used:

- A rewriting strategy (TL_recon) that is used to implement functions such as gmap and updateSalary.

- A reduction strategy (TL_red) that is used to implement selectSalary.

- A reduction strategy that also provides access to constructor names (TL_red_con). This strategy is used to implement gshow.

- A twin traversal strategy (TL_red_lockstep) that is used to implement functions with multiple arguments such as equality.

- A shallow reduction traversal ( $T L_{-} r$ red_shallow) that is used to implement gmapQ.

- A couple of reduction traversals that abstract over $\star \rightarrow \star$-types ( TL_red_cr1) and $\star \rightarrow \star \rightarrow \star$-types $\left(T L \_r e d \_c r 2\right)$.

- a traversal strategy for producer functions.

\subsection{Uniplate}

The Uniplate library provides a form of generic programming based on traversal combinators. There are two sorts of traversals: single type and multi-type traversals. Unlike SYB, Uniplate combinators do not require a type system that supports rank-2 types. This is because traversals are customized by functions that are monomorphic rather than polymorphic, as in SYB. An example of a Uniplate combinator is the bottom-up transformation traversal (transform).

$$
\text { transform }:: \text { Uniplate } \mathrm{a} \Rightarrow(\mathrm{a} \rightarrow \mathrm{a}) \rightarrow \mathrm{a} \rightarrow \mathrm{a}
$$

The transform traversal applies the function argument to every avalue that is contained within the a argument. The Uniplate type class is the analog of Data in SYB, it provides a set of common traversals on top of which more sophisticated traversals are defined. The Uniplate type class is equipped with 10 traversal methods. However, all of them can be defined in terms of the fundamental uniplate operation (the analog in SYB is gfoldl):

$$
\text { uniplate }:: \text { Uniplate } \mathrm{a} \Rightarrow \mathrm{a} \rightarrow([\mathrm{a}],[\mathrm{a}] \rightarrow \mathrm{a})
$$

This function takes an argument of type a and returns a pair of (1) a list of maximal substructures with type a, and (2) a function that rebuilds the argument using new values for those substructures. For example, uniplate (Bin (Leaf 1) (Leaf 2)) yields ([Leaf 1, Leaf 2], Bin). The uniplate function can be seen as the structure representation of a-values, because all combinator definitions ultimately rely on it.

In Uniplate, the arguments of traversals cannot perform case analysis on types, because they are monomorphic functions. Interestingly, this is not a limitation in practice, because in general the interesting case of a traversal is restricted to one type. This is also the case of functions selectSalary and updateSalary in our suite.

Uniplate also provides multi-type traversals using multi-parameter type classes. Consider the multi-type variant of transform:

$$
\text { transformBi :: Biplate } \mathrm{b} \text { a } \Rightarrow(\mathrm{a} \rightarrow \mathrm{a}) \rightarrow \mathrm{b} \rightarrow \mathrm{b}
$$

This combinator applies the function argument to all a-values that are contained in the $b$ argument. For example, this is how Uniplate implements updateSalary:

$$
\begin{aligned}
& \text { increase }:: \text { Float } \rightarrow \text { Company } \rightarrow \text { Company } \\
& \text { increase } k=\text { transformBi }(\text { inc } S k) \\
& \text { inc } S:: \text { Float } \rightarrow \text { Salary } \rightarrow \text { Salary } \\
& \text { incS } k(S s)=S(s \star(1+k))
\end{aligned}
$$

Multi-type traversals are more flexible than single-type traversals, in that they allow the specification of an ad-hoc case on one type while doing the traversal on another.

\subsection{Detailed evaluation}

The evaluation is described criterion by criterion below and summarised in Fig. 9.

Universe Size. What are the types that a generic function can be used on? That is, what are the datatypes to which generic universe extension is possible? This question is answered separately for each of the sub-criteria of universe size.

A library scores good on regular, higher-kinded datatypes, nested datatypes, higher-kinded and nested datatypes, and other Haskell 98 datatypes, if it can generically extend the universe to BinTree, GRose, Perfect, NGRose, and Company respectively, and apply generic equality to them. The library scores bad otherwise.

The LIGD approach can represent the structure of all datatypes in the universe size test, therefore it scores good on this criterion. The structure of a datatype $\mathrm{T}$ of kind $\star$ is represented as a Rep $\mathrm{T}$ value constructed with RType. For instance, the datatype Company is represented by rCompany, which has type Rep Company. Type constructors are represented by functions on representations. For instance, lists are represented by rList which has type Rep a $\rightarrow \operatorname{Rep}[a]$. The encoding can be generalised to higher-kinded parameters and nested datatypes: they are represented by higher-order functions with rank-2 types. 
The PolyLib library is limited to regular datatypes (with one parameter) and cannot handle mutually recursive datatypes, so the set of datatypes (the universe) supported is relatively small.

The SYB library scores well on the universe size criteria, even for the Perfect datatype, which is nested. The GRose datatype presents difficulties because it has an type argument of kind $\star \rightarrow \star$, and so instances for Data and Typeable cannot be automatically derived. However, these instances can be written by the programmer, therefore generic universe extension to GRose is supported. Note that in the instance for GRose

$$
\begin{gathered}
\text { instance (Typeable } 1 \mathrm{f} \text {, Typeable a, Data a } \\
\text {, Data }(\mathrm{f} \text { (GRose } \mathrm{f} \text { a)) }) \\
\Rightarrow \text { Data (GRose } \mathrm{f} \mathrm{a} \text { ) where ... }
\end{gathered}
$$

the instance head (GRose $f$ a) reappears in the context. This implies that cycle-aware constraint resolution [Lämmel and Peyton Jones, 2005] is required to type this program. In contrast, SYB does not support generic extension to NGRose. The reason is that in the NGRose instance

$$
\begin{gathered}
\text { instance (Typeable1 } \mathrm{f} \text {, Typeable a, Data a } \\
\text {, Data }(\mathrm{f}(\text { NGRose }(\text { Comp } \mathrm{f} f) \mathrm{a}))) \\
\Rightarrow \text { Data (NGRose } \mathrm{f} \text { a) where ... }
\end{gathered}
$$

the head (NGRose $f$ a) becomes bigger in the context, namely $f$ becomes Comp $f f$, and therefore cycle-aware resolution is not enough to type check programs using this instance.

The universe of SYB3 is even smaller than that of SYB: Perfect is not supported. The instance that is automatically derived looks as follows:

$$
\begin{gathered}
\text { instance (Data ctx }(\text { Perfect }(\text { Fork a }))), \ldots) \Rightarrow \\
\text { Data ctx (Perfect a) where }
\end{gathered}
$$

Note that this instance has the same problems as the NGRose instance in SYB. Programs that use it, will not type check. The NGRose datatype is not supported for the same reason. Universe extension for the BinTree datatype is supported, but we surprisingly have to manually write a Data instance for it. The reason is that Derive, the module that automatically generates representations, produces an erroneous Data instance. Indeed, the generated instance causes non-termination at runtime. The reason, we believe, is an erroneous Typeable dictionary at runtime, which causes looping when it is used to cast inside generic equality. We give BinTree a good score anyway, because this is a problem of Derive, rather than of support for regular datatypes.

The Spine approach has the advantage of a large universe size: it can handle all datatypes in the universe size test.

In EMGM, the structure of a datatype $T$ is represented by a value of type Generic $g \Rightarrow g \mathrm{~T}$, which is built using the view method. Like in LIGD, type constructors are encoded as functions over representations, for example the type constructor list is encoded as Generic $\mathrm{g} \Rightarrow \mathrm{g}$ a $\rightarrow \mathrm{g}$ [a]. Higher-kinded datatypes such as GRose and even NGRose can also be encoded in EMGM. But NGRose cannot be used with implicit representations, because the type class that implements them (GRep), would need an instance that raises the same issues as the Data instance above. In summary, generic extension to NGRose is supported but at the cost of reduced functionality. Therefore EMGM scores good for all criteria, except for nested and higher kinded datatypes where it scores sufficient.

The RepLib library cannot represent datatypes with higherkinded arguments. It follows that it satisfies the tests for regular and other Haskell 98 datatypes only.

The Smash library represents the structure of all datatypes in the universe size test. Nested datatypes such as Perfect and NGRose present problems similar to those in other type class based ap- proaches. However, it is possible to represent them with some effort. The Company datatype caused looping during type checking. A workaround is possible, but at the moment we have not identified the exact cause of the problem. Because of the difficulties mentioned, Smash scores sufficient on the problematic sub-criteria.

The Uniplate library scores well on universe size, it can handle all datatypes except nested generalised rose trees. To support higher-kinded datatypes, the same instances for Data and Typeable are used as for SYB.

Subuniverses. Is it possible to restrict the use of a generic function on a particular set of datatypes? An approach scores good if uses of the generic function on datatypes outside of the set are flagged as compile-time errors.

The RepLib and EMGM approaches score good on this criterion. In both approaches the set of types to which a generic function can be instantiated is controlled by instance declarations. For example, if generic equality is to be used on lists, the programmer is expected to write the following instance (or an instance containing an ad-hoc definition):

\section{instance GenericList Geq}

otherwise compilation fails with a type checking error when applying equality to lists.

PolyLib also supports subuniverses - a TIF is limited to the instances defined and this is compiler checked.

First-class generic functions. Can a generic function take a generic function as an argument? If $\operatorname{gmap} Q$ can be implemented in a library such that it can be passed a generic function (for example, gshow) as argument, the library scores good. If gmap $Q$ can be written but at the price of additional complexity the library scores sufficient. Otherwise, if gmap $Q$ cannot be implemented, the library scores bad.

In LIGD, SYB, and Spine, a generic function is a polymorphic Haskell function, so it is a first-class value in Haskell implementations that support rank-2 polymorphism. Consider for example gmap $Q$ in LIGD:

$$
\text { gmap } Q::(\forall \mathrm{a} . \operatorname{Rep} \mathrm{a} \rightarrow \mathrm{a} \rightarrow \mathrm{r}) \rightarrow \operatorname{Rep} \mathrm{b} \rightarrow \mathrm{b} \rightarrow[\mathrm{r}]
$$

Here the function argument is polymorphic, which allows gmap $Q$ to instantiate it to different types. In short, in LIGD, SYB, and Spine the generic function argument is just a normal functional argument, albeit a polymorphic one. It follows that LIGD, SYB, and Spine score good on this criterion.

EMGM scores sufficient because although EMGM supports first-class generic functions, they are implemented in a rather complicated way. The reason is that the type class system needs to track calls to generic functions. So we are forced to go from a relatively simple (but wrong) signature for GMapQ:

$$
\begin{aligned}
& \text { data GMapQ a }=G M a p Q\{ \\
& \quad \operatorname{gmap} Q::(\ldots \rightarrow \mathrm{r}) \rightarrow \mathrm{a} \rightarrow[\mathrm{r}]\}
\end{aligned}
$$

to a type signature that allows to track calls to the generic function argument. The new signature below abstracts over a type g, the signature of the function argument, and $\mathrm{garg}$, which is the generic function argument itself.

$$
\begin{aligned}
& \text { data GMapQ g a }=G M a p Q\{ \\
& \text { garg }:: \mathrm{g} \text { a, } \\
& \operatorname{gmap} Q::(\forall \mathrm{a} \cdot \mathrm{g} \text { a } \rightarrow \mathrm{a} \rightarrow \mathrm{r}) \rightarrow \mathrm{a} \rightarrow[\mathrm{r}]\}
\end{aligned}
$$

This makes the definition of $\operatorname{gmap} Q$, significantly more complex than other functions, such as generic equality.

The test function $g$ map $Q$ can be defined with no difficulties in SYB3 and RepLib, which therefore score good.

Like in SYB, generic programming in Uniplate is combinator based. However, combinators are parametrised over monomorphic 
functions and not over other generic functions, as is the case in SYB. It is not evident how gmap $Q$ would be implemented in Uniplate, hence it scores bad.

In Smash, gmapQ is implemented using the TL_red_shallow reduction strategy. However, having a new strategy altogether, in place of using an existing one, imposes the burden of one more structure representation for the user. Therefore this library only scores sufficient.

Abstraction over type constructors. Is a generic library able to define the generic functions gmap and crushRight? If a library can define both functions which can then be instantiated to mapBinTree and flatten WTree, which have types:

$$
\begin{aligned}
& \text { mapBinTree }::(\mathrm{a} \rightarrow \mathrm{b}) \rightarrow \text { BinTree } \mathrm{a} \rightarrow \text { BinTree } \mathrm{b} \\
& \text { flatten WTree }:: \text { WTree a } \mathrm{w} \rightarrow[\mathrm{a}]
\end{aligned}
$$

then the approach scores good. If the library can only support the definition of one of the functions or none, it scores sufficient or bad, respectively.

The LIGD, EMGM, and RepLib libraries support the definitions of gmap and crushRight by means of arity-based type representations (Section 3.3.2), and their instantiations yield functions mapBin Tree and flatten WTree as required. Hence these libraries score good.

PolyLib includes the definition of gmap and crushRight. However these functions can be instantiated only to regular datatypes, which have kind $\star \rightarrow \star$. If follows that flatten WTree cannot be obtained from crushRight because WTree has kind $\star \rightarrow \star \rightarrow \star$. Therefore PolyLib scores sufficient.

In Smash, the definition of gmap and crushRight are supported. Generic map is implemented by means of the rewriting traversal strategy $T L_{-}$recon. This strategy supports ad-hoc cases that can change the type of elements, so gmap can be instantiated to mapBinTree. The definition of crushRight uses two special purpose reduction strategies, one for $\star \rightarrow \star$-types and the other for $\star \rightarrow \star \rightarrow \star$-types.

The SYB, Uniplate, and SYB3 libraries represent types with kind $\star$ but they do not represent type constructors. It follows that they are unable to support the definitions of gmap and crushRight.

Separate compilation. Is generic universe extension modular? Approaches that can instantiate generic equality to BinTree without recompiling the function definition or the type/structure representation score good.

The LIGD, EMGM, and RepLib libraries score good on this criterion. In LIGD and RepLib, representation types have a constructor to represent the structure of datatypes, namely RType and Data1. It follows that generic universe extension requires no extension of the representation datatypes and therefore no recompilation. In EMGM, datatype structure is represented by view, so a similar argument applies.

PolyLib uses instance declarations (of the FunctorOf class) for universe extension, so the score is good.

The SYB, Uniplate, and SYB3 libraries score good on this criterion. Generic universe extension is achieved by defining Data and Typeable instances for BinTree, which does not require recompilation of existing code in other modules. In Smash, the universe is likewise extended by defining instances (of LDat).

The Spine library scores bad on this criterion. The reason is that universe extension requires that the datatype, in this case BinTree, is represented by a constructor in the GADT that encodes types. Because this datatype is defined in a separate module, recompilation is required.

Ad-hoc definitions for datatypes. Can a generic function contain specific behaviour for a particular datatype, and let the remaining datatypes be handled generically? Moreover, the use of ad-hoc cases should not require recompilation of existing code (for instance the type representations). If the function selectSalary can be implemented by a library using an ad-hoc case for the Salary datatype, it scores good. Otherwise, it scores bad.

In LIGD and Spine, the ad-hoc case in the selectSalary function would have to be given by pattern matching on the type representation constructor that encodes Salary, namely RSalary. However, this requires the type representation datatype to be extended, and hence the recompilation of the module that contains it. For this reason both approaches score bad.

In PolyLib, the Company datatypes cannot be represented (only regular datatypes are supported), so the selectSalary test cannot be compiled. But ad-hoc cases is supported for regular datatypes, so PolyLib scores sufficient.

In $S Y B$, ad-hoc cases for queries are supported by means of the $m k Q$ and ext $Q$ combinators. Such combinators are also available for other traversals, for example transformations. The only requirement for ad-hoc cases is that the type being case-analysed should be an instance of the Typeable type class. The new instance does not require recompilation of other modules, so SYB scores good on this criterion.

The SYB3, EMGM, and RepLib libraries score good on this criterion. In SYB3 and RepLib, ad-hoc cases are given as an instance of the type class that implements the generic function. In EMGM, ad-hoc cases are given as an instance of Generic (or a subclass corresponding to the represented datatype). Because ad-hoc cases are given as type class instances, recompilation is not needed.

In Uniplate, it is possible to define datatype specific behaviour for a multi-type traversal. This is usually achieved by using a traversal combinator that is parametrised over (1) the type on which the traversal is performed, (2) the type for which the ad-hoc case is given, and (3) the ad-hoc case function. Function transform $B i$ is such a combinator.

In Smash, a monomorphic ad-hoc definition is given as an element in the list of ad-hoc cases (a function of type ad_hoc_type $\rightarrow$ String in case of gshow). Smash performs case analysis on types using a type equality operation implemented as a type class, which was originally used to implement extensible records [Kiselyov et al., 2004]. Because no recompilation of the library modules is needed to allow case analysis over a new type, this library scores good on this criterion.

Ad-hoc definitions for constructors. Can we give an ad-hoc definition for a particular constructor, and let the remaining constructors be handled generically? We take the function rmWeights as our test. If a library allows the implementation of this function such that an explicit case for the WithWeight constructor is given and the remaining constructors are handled generically, then that library scores good on this criterion.

The LIGD and Spine libraries do not support ad-hoc definitions for datatypes. It follows that trying to implement such definitions for constructors would require recompilation (because ad-hoc definitions would be needed). Should we then declare that this criterion is unsupported? We do not think so. The user might be interested in providing an ad-hoc constructor definition, and still be willing to pay the price of lack of support for ad-hoc definitions for datatypes. We make this explicit in Figure 8: it is allowed to cheat in the rmWeights test for the ad-hoc definitions for datatypes criterion.

The LIGD and Spine libraries are able to support the definition of $r m$ Weights as required. The Spine library scores good, but LIGD scores sufficient because of additional complications. As explained in Section 3.2.3, LIGD needs a modified datatype representation that allows ad-hoc definitions. This gives a total of two representations for WTree, one for generic extension and the other for non-generic extension. 
PolyLib does not support ad hoc definitions for constructors.

The six approaches that support ad-hoc definitions for datatypes, also support ad-hoc definitions for constructors, hence SYB, SYB3, Uniplate, EMGM, Smash, and RepLib score good on this criterion.

Extensibility. Can a programmer extend the universe of a generic function in a different module without the need for recompilation? Libraries that allow the extension of the generic gshow function with a case for printing lists score good. As mentioned before, extensibility is not possible for approaches that do not support adhoc cases. For this reason the LIGD and Spine approaches score bad.

Before proceeding to the evaluation, let us remark that a library that supports ad-hoc cases can be made extensible. The trick is to extend the generic function with an argument that receives the adhoc case (or cases) with which the generic function is extended. Such a trick would be possible with SYB and Smash, for example. However, this is unacceptable for two reasons. First, this would impose a burden on the user: the generic function has to be "closed" by the programmer before use. Second, functionality that is implemented on top of such an extensible generic function would have to expose the extension argument in its interface. An example of such functionality is discussed by Lämmel and Peyton Jones [2005] in their QuickCheck case study. QuickCheck implements shrinking of test data by using a shrink generic function, which should be extensible. If function extensibility would be implemented as proposed in this paragraph, the high-level quickCheck function would have to include extension arguments for shrink. For this reason, we do not accept such implementations of extensibility in our evaluation.

PolyLib is extensible because it uses class instances to extend the universe. However, it scores sufficient because Company, the datatype that is used in this test, is not supported by PolyLib.

The SYB, Smash, and Uniplate libraries support ad-hoc definitions, but do not support extensible generic functions. Therefore they score bad on this criterion.

In SYB3, EMGM, and RepLib, ad-hoc cases are given in instance declarations, which can reside in separate modules. Therefore these libraries support extensible generic functions.

Multiple arguments. Can a generic programming library support a generic function definition that consumes more than one generic argument, such as the generic equality function? If generic equality is definable then the approach scores good. If the definition is more involved than those of other consumer functions such as gshow and selectSalary, then the approach scores sufficient.

The LIGD, PolyLib, EMGM, and RepLib approaches support the definition of generic equality. Furthermore, equality is not more difficult to define than other consumer functions. For example, in LIGD, every case of equality matches a type representation and two structurally represented values that are to be compared. Because these two values have the same type, usual pattern matching is enough to give the definition. Defining generic equality is not any more difficult than defining gshow. Therefore, these approaches score good on this criterion.

In Spine, the generic equality function is defined as follows:

$$
\begin{aligned}
& \text { equal }:: \text { Typed a } \rightarrow \text { Typed } \mathrm{b} \rightarrow \text { Bool } \\
& \text { equal } x_{1} x_{2}=\text { equalSpines }\left(\text { toSpine } x_{1}\right)\left(\text { toSpine } x_{2}\right)
\end{aligned}
$$

It converts both values to their Spine representation and the real comparison is performed on the spine itself:

$$
\begin{aligned}
\text { equalSpines }:: \text { Spine } \mathrm{a} \rightarrow \text { Spine } \mathrm{b} \rightarrow \text { Bool } \\
\text { equalSpines }\left(\text { Con } c_{1}\right)\left(\text { Con } c_{2}\right)=\text { name } c_{1}==\text { name } c_{2} \\
\text { equalSpines }\left(f_{1}: \$ x_{1}\right)\left(f_{2}: \$ x_{2}\right)=\text { equalSpines } f_{1} f_{2} \wedge \\
\\
\text { equal } x_{1} x_{2} \\
\text { equalSpines }-\quad-\quad \text { False }
\end{aligned}
$$

This function is not more complicated than other consumer functions in this approach. Therefore Spine scores good. It can be argued, however, that this function is not entirely satisfactory. Equality relies ultimately on equality of constructor names, therefore the user could make a mistake when providing a constructor name in the representation.

The SYB library only scores sufficient on this criterion. The reason is that the definition is not as direct as for other functions such as gshow and selectSalary. While the Spine definition equalSpine can inspect the two arguments to be compared, in SYB the gfoldl combinator forces to process one argument at a time. For this reason, the definition of generic equality has to perform the traversal of the arguments in two stages. The definition can be found in Lämmel and Peyton Jones [2004]

Smash supports multiple arguments to a generic function essentially through currying - a special traversal strategy that traverses several terms in lockstep. However, the fact that a special purpose traversal must be used for functions on multiple arguments imposes a burden on the user. The user has to write one more structure representation per datatype. Therefore Smash only scores sufficient.

The traversal combinators of the Uniplate library are designed for single argument consumers. We have not been able to write a generic equality function for this approach, so Uniplate scores bad.

Constructor names. Is the approach able to provide the names of the constructors to which the generic function is applied? Library approaches that support the definition of gshow score good.

With the exception of Uniplate, all generic programming libraries discussed in this paper provide support for constructor names in their structure representations. The Uniplate library itself does not provide any means to access constructor names.

Consumers, transformers, and producers. Generic libraries that can define functions in the three categories: consumers, transformers and producers, score good. This is the case for LIGD, PolyLib, EMGM, and RepLib.

If a library uses a different structure representation or type representation for say consumer and producer functions, that library scores sufficient. This is the case of SYB, SYB3, Smash, and Spine. Furthermore, Smash uses a different representation (traversal strategy) for transformers than for consumers, therefore it scores sufficient as well on that criterion. Why is it a disadvantage to have several structure representations? Because this implies more work for the programmer when doing generic universe extension: more representations have to be written per datatype.

The Uniplate library does not contain combinators to write producer functions, so it scores bad.

Performance. We have used some of the test functions for a performance benchmark but the results are very sensitive to small code differences and compiler optimizations so firm conclusions are difficult to draw. As a sample, Figure 10 shows running times (in multiples of the running time of a hand-coded monomorphic version) for the $g e q$, selectInt and $r m W e i g h t s$ tests.

For the $g e q$ test, the compiler manages to produce very efficient code for EMGM, while the SYB family has problems with the two-argument traversal. The fact that the overhead for $\mathrm{rmWeights}$ starts around a factor of two is disappointing, but shows the improvement potential from using partial evaluation techniques (as used in C++ STL). The best overall performance score is shared between EMGM, Smash and Uniplate.

Portability. Figure 11 shows that the majority of approaches rely on compiler extensions provided by GHC to some extent. Approaches that are most portable rely on few extensions or on extensions that are likely to be included in Haskell prime [Haskell Prime list, 2006]. 


\begin{tabular}{c|ccccccccc} 
Test & LIGD & PolyLib & SYB & SYB3 & Spine & EMGM & RepLib & Smash & Uniplate \\
\hline geq & 26 & 8 & 52 & 70 & 13 & 1.5 & 8 & 4 & - \\
selectInt & 3 & - & 5 & 2 & 2 & 3 & 2 & 2 & 0.8 \\
rmWeights & 3 & - & 78 & 7 & 2 & 4 & 4 & 2 & 4
\end{tabular}

Figure 10. Preliminary performance experiments.

Of all generic programming libraries, LIGD, EMGM, and Uniplate are the most portable. The only compiler extension that LIGD relies on is existential types, and this extension is very likely to be included in the next Haskell standard [Haskell Prime list, 2006]. However, LIGD needs rank- 2 types for the representations of GRose and NGRose, but not for other representations or functions. Hence rank-2 types are not an essential part of the LIGD approach.

The EMGM library relies on multi-parameter type classes (also likely to be included in the next standard) to implement implicit type representations. This type class makes EMGM more convenient to use, but, even without it, it would still be possible to do generic programming in EMGM, be in a less convenient way.

In SYB3, overlapping and undecidable instances are needed for the implementation of extensibility and ad-hoc cases. Overlapping instances arise because of the overlap between the generic case and the type-specific cases. Undecidable instances are required for the Sat type class, which is used to implement abstraction over type classes

Rank-w polymorphism is required to type the gfoldl and gunfoldl combinators in SYB and SYB3.

GADTs are used by the Spine and RepLib libraries to represent types and their structure. Both libraries also use existential types in their representations.

The unsafeCoerce extension is used to implement type safe casts in SYB, SYB3, and RepLib.

The RepLib and SYB3 libraries provide automatic generation of representations, which is implemented using Template Haskell. The SYB library, on the other hand, relies on compiler support for deriving Data and Typeable instances.

Uniplate can be defined in Haskell 98. However, in order to use multi-type traversals, multi-parameter type classes are needed. Uniplate can derive representations by either using a tool that uses Template Haskell, or by relying on compiler support to derive Data and Typeable. However, the use of these extension is optional, because structure representations can be written by programmers directly.

Smash relies on various extensions such as multi-parameter type classes, undecidable instances, overlapping instances, and functional dependencies. This is needed to implement the techniques for handling ad-hoc cases and traversal strategies.

Overhead of library use. How much overhead is imposed on the programmer for the use of a generic programming library? We are interested in the following aspects:

- Automatic generation of representations. The three libraries that offer support for automatic generation of representations are SYB, SYB3, Uniplate, and RepLib.

The SYB library relies on GHC to generate Typeable and Data instances for new datatypes. The SYB library scores good on this criterion.

The RepLib library includes Template Haskell code to generate structure representations for new datatypes in its distribution. However, RepLib does not support the generation of representations for arity two generic functions and does not include the machinery for such representations. Furthermore automatic generation fails when a type synonym is used in a datatype declaration. RepLib scores sufficient because of the problems mentioned.

The SYB3 library also uses Template Haskell to generate representations. However, the generated representations for BinTree cause non-termination when type-safe casts are used on a BinTree value. This is a serious problem: regular datatypes and type-safe casting are very commonly used. Hence this approach does not score good but sufficient.

Uniplate can use the Typeable and Data instances of GHC for automatic generation of representations. Furthermore, a separate tool, based on Template Haskell, is provided to derive instances. The Uniplate library scores good on this criterion.

PolyLib used to include support for generation of representations, but this functionality broke with version 2 of Template Haskell.

Note that automatic generation of representations in all approaches is limited to datatypes with no arguments of higherkinds, hence GRose and NGRose are not supported.

- Number of structure representations. PolyLib only supports regular datatypes of arity one, thus it only has one representation. It would be straightforward to add a new representation for each arity $a$, but it would still only support regular datatypes.

The LIGD, EMGM, and RepLib libraries have two sorts of representations: (1) a representation for $\star$-types (for example Rep in Section 2), and (2) representations for type constructors, which are arity-based (Section 3.3.2). The latter consists of a number of arity-specific representations. For example, to write the gmap function we would have to use a representation of arity two. Which arities should be supported? Probably the best answer is to support the arities for which useful generic functions are known: crush (arity one), gmap (arity two), and generic zip (arity three). This makes a total of four representations needed for these libraries, one to represent $\star$-types, and three for all useful arities.

Note, however, that functions of arity one can be defined using a representation of arity three. This means that we could remove representations of arities one and two. So, we could imagine a library needing only two representations. The next step is to subsume the representation for $\star$-types with the arity three representation. Although this makes some approaches more inconvenient - for instance, the EMGM approach would lose the generic dispatcher. Although reducing the number of representations is possible, we do not do so in this comparison, because it is rather inelegant. Defining generic equality using a representation of arity three would leave a number of unused type variables that might make the definition confusing.

When a new datatype is used with SYB/SYB3, the structure representation is given in a Data instance. This instance has two methods gfoldl and gunfoldl which are used for consumer and transformer, and producer generic functions respectively. Therefore every new datatype needs two representations to be used with SYB/SYB3 functions. 
The Spine library needs three structure representations per datatype. The first, the spine representation, is used with consumer and transformer functions. And the second, the type spine view, is used with producer functions. The third representation is used to write generic functions that abstract over type constructors of kind $\star \rightarrow \star$.

In Uniplate, the number of structure representations that are needed can range from one Uniplate instance per datatype, to $O\left(n^{2}\right)$ instances for a system of $n$ datatypes, when maximum performance is wanted. For our comparison, we assume that one representation is needed.

Smash is specifically designed to allow arbitrary number of custom traversal strategies. Although three strategies are fundamental - reconstruction, reduction, and parallel traversal the simplified variations of these turn out more convenient and frequently used. However, this also means that the programmer defines more structure representations than in other libraries. The representations that are used in this evaluation are eight: a rewriting strategy, a reduction strategy, a reduction strategy with constructor names, a twin traversal strategy, a shallow reduction traversal, two traversals for abstracting over type constructors of kinds $\star \rightarrow \star$ and $\star \rightarrow \star \rightarrow \star$, and a traversal strategy for producer functions.

- Work to instantiate a generic function. The instantiation of a generic function requires a value representing the type to which it is instantiated. The representation can be explicit (the programmer has to supply it) or implicit. Approaches that use type classes can make representations implicit, making instantiation easier for the user. This is the case of SYB, SYB3, EMGM, RepLib, and Smash.

However, some type-class based approaches, such as EMGM and RepLib, require an instance declaration per function per datatype in order to allow instantiation of the function to that datatype. This requires additional work from the user, but on the other hand this enables control over the instantiation of a function (subuniverses).

The SYB, Smash, Uniplate, and SYB3 libraries have implicit representation arguments and do not need instance declarations per function per type, therefore these libraries score good.

- Work to define a generic function. Is it possible to quickly write a simple generic function? A library scores good if it requires roughly one definition per generic function case, with no need for additional artefacts.

The LIGD, SYB3, and RepLib libraries score bad on this criterion. In LIGD, the definitions of generic functions become more verbose because of the emulation of GADTs. However, this overhead does not arise in implementations of LIGD that use GADTs directly. In RepLib we need to define a dictionary datatype, and an instance of the Sat type class, in addition to the type class definition that implements the generic function. In SYB3 the definitions needed (besides a type class for the function) are the dictionary type, a Sat instance for the dictionary, and a dictionary proxy value. Therefore these libraries only score sufficient. The other libraries score good because they demand less "encoding work" when defining a generic function.

Practical aspects. For this criterion we consider aspects such as

- there is a library distribution available online,

- the library interface is well-documented,

- and other aspects such as an interface organized into modules, and common generic functions.
The LIGD, EMGM, and Spine libraries do not have distributions online. These libraries score bad.

PolyLib has an online distribution (as part of PolyP version 2) but the library is not maintained anymore.

The SYB library is distributed with the GHC compiler. This distribution includes a number of traversal combinators for common generic programming tasks and Haddock documentation. The GHC compiler supports the automatic generation of Typeable and Data instances. This library scores good.

The official SYB3 distribution failed to compile with the versions of GHC that we used in this comparison $(6.6,6.8 .1,6.8 .2)$. This distribution can be downloaded from: http://homepages . cwi.nl/ ralf/syb3/code.html. There is an alternative distribution of this library which is available as a Darcs repository from: http://happs.org/HAppS/syb-with-class. This distribution is a cabal package that includes Hadock documentation for the functions that it provides. However, it does not contain many important combinators such as gmapAccum $Q$ and gzipWith $Q$ amongst others.

The RepLib library has an online distribution. The distribution includes a number of pre-defined generic functions, the structure representation generation machinery and Haddock documentation. The score of this library is good.

The Uniplate library and haddock documentation are available online (also via HackageDB). Just like SYB it can derive representations. This library scores good.

The Smash library is distributed as a standalone module that can be downloaded from http://okmij.org/ftp/Haskell/ generics.html\#Smash. There are a few example functions in that module that illustrate the use of the approach. However, the library is not as organized as SYB3, and therefore it scores bad.

Implementation mechanisms. What are the mechanisms through which a library encodes a type or its representation? We have the following options:

- Types and structure represented by GADTs. Types are encoded by a representation GADT, where each type is represented by a constructor. The GADT may also have a constructor which encodes datatypes structurally (like RType in this paper).

- Types and structure represented by datatypes. When GADTs are not available, it is still possible to emulate them by embedding conversion functions in the datatype constructors. In this way a normal datatype can represent types and their structure.

- Rank-2 structure representation combinators. Yet another alternative is to represent the structure of a datatype using a rank-2 combinator such as gfoldl in SYB.

- Type safe cast. Type safe casts are used to implement typespecific functionality, or ad-hoc cases. Type safe casting converts a-values into $b$-values at runtime, where $a$ and $b$ are unknown statically.

- Extensible records and type-level evaluation. The work of Kiselyov et al. [2004] introduces various techniques to implement extensible records in Haskell. The techniques make advanced use of type classes to perform record lookup statically. This operation is an instance of a general design pattern: encoding type-level computations using multi-parameter type classes and functional dependencies.

- Type classes. Type classes may be used in a variety of ways: to represent types and their structure and perform case analysis on them, to overload structure representation combinators, and to provide extensibility to generic functions.

- Abstraction over type classes. Generic programming libraries that support extensible generic functions, do so by using type 


\begin{tabular}{|c|c|c|c|c|c|c|c|c|c|}
\hline & LIGD & PolyLib & SYB & SYB3 & Spine & EMGM & RepLib & Smash & Uniplate \\
\hline $\begin{array}{l}\text { Implementation mechanisms } \\
\text { Type representation is GADT } \\
\text { Type representation is D.T. } \\
\text { Rank-2 struc. repr. combinator } \\
\text { Type safe cast } \\
\text { Extensible rec. and type eval. } \\
\text { Type classes } \\
\text { Abstraction over type classes }\end{array}$ & & & & & & 0 & 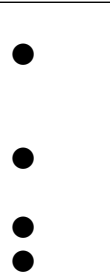 & 0 & T \\
\hline $\begin{array}{l}\text { Compiler extensions } \\
\text { Undecidable instances } \\
\text { Overlapping instances } \\
\text { Multi-parameter typeclasses } \\
\text { Functional dependencies } \\
\text { Rank-2 polymorphism } \\
\text { Existential types } \\
\text { GADTs } \\
\text { unsafeCoerce } \\
\text { Template Haskell } \\
\text { Derive Data and Typeable }\end{array}$ & & & & & & & & 0 & 0 \\
\hline $\begin{array}{l}\text { Views } \\
\text { Fixed point view } \\
\text { Sum of products } \\
\text { Spine } \\
\text { Lifted spine } \\
\text { RepLib } \\
\text { Uniplate } \\
\text { Smash }\end{array}$ & & & & & & & & & \\
\hline
\end{tabular}

Figure 11. Implementation mechanisms, required compiler extensions, and views.

classes. Some of this approaches, however, require a form of abstraction over type classes, which can be encoded by a technique that uses explicit dictionaries, popularized by Lämmel and Peyton Jones [2005].

The LIGD and Spine libraries represent types and structure as datatypes and GADTs respectively, while EMGM uses type classes to do so.

In SYB and SYB3, datatypes are structurally represented by rank-2 combinators gfoldl and gunfoldl.

Ad-hoc cases in SYB are given using pre-defined combinators such as $\operatorname{ext} Q$ and $m k Q$, which are implemented using type safe casts.

In SYB3, case analysis over types is performed directly by the type class system, because generic functions are type classes. However, type safe casts are still important because they are used to implement functions such as equality. Furthermore, this approach implements abstraction over type classes to support extensibility.

The RepLib library is an interesting combination. It has a datatype that represents types and their structure, and so generic functions are defined by pattern matching on those representation values. On the other hand, RepLib also uses type classes to allow the extension of a generic function with a new type-specific case. To allow extension type classes must encode type class abstraction using the same technique as in SYB3. Optionally, the RepLib library allows the programmer to use a programming style reminiscent of SYB, where ad-hoc cases are aggregated using functions such as $e x t Q$ and $m k Q$. These combinators are implemented using the GADT-based representations and type safe casts.

The Smash library uses an extensible record-like list of functions to encode ad-hoc cases. Case analysis on types is performed by a lookup operation, which in turn is implemented by type-level typecase. This library also uses type-level evaluation to determine the argument and return types of method gapp, based on the traversal strategy and the list of ad-hoc cases.

Views. What are the views that the generic library supports? That is, how are datatypes encoded in structure representations and what are the view types used in them?

The LIGD and EMGM libraries encode datatypes as sums of products, where the sums encode the choice of a constructor and the products encode the fields used in them. This view is usually referred to as sum of products. The representations for sums of products that are based on arities (Section 3.3.2) can be used to abstract over type constructors.

The Spine approach uses the Spine datatype to make the application of a constructor to its arguments observable to a generic function. As observed by the authors of this view, the gfoldl combinator used in SYB and SYB3 is a catamorphism of a Spine value, and hence these approaches also use the spine view. The SYB, SYB3, and Spine approaches also provide a type-spine view that is used to write producer generic functions. Unlike SYB and SYB3, Spine supports abstraction over $\star \rightarrow \star$-types due to an additional view called the lifted Spine view.

The RepLib library also has a different view, datatypes are represented as a list of constructor representations, which are a heterogeneous list of constructor arguments. Like in LIGD and EMGM, the structure representation can be adapted to be arity based, in order to support abstraction over type constructors.

The Uniplate library also has a view of its own. All traversal combinators in this library are based on the uniplate operation. This operation takes an argument of a particular datatype, and returns its children that have the same type as the argument, and a function to reconstruct the argument with new children.

It is difficult to point to specific views in the Smash library. Although there are three basic strategies (rewriting, reduction, and 
lockstep reduction), the rest of the strategies are not defined using any of the more fundamental ones. Hence every traversal strategy can be considered as a way to view the structure of a datatype.

Ease of learning and use. It is hard to determine how easy it is to learn how to use a library. We approximate this criterion by looking at the mechanisms used in the implementation of the libraries. Below we describe the difficulties that arise with some of the implementation mechanisms:

- Rank-2 structure representation combinators. There are two problems with rank-2 structure representation combinators. First, rank-2 polymorphic types are difficult to understand for beginning users. This implies that defining a generic function from scratch - that is, using the type structure directly, bypassing any pre-defined combinators - presents more difficulties than in other approaches. Second, structure representation combinators such as those used in SYB can be used directly to define functions that consume one argument. But if two arguments are to be consumed instead (which is the case in generic equality), then the definition of the function becomes complicated.

- Extensible records and type-level evaluation. The techniques to encode extensible records make advanced use of type classes and functional dependencies. This encoding can be troublesome to the beginning generic programmer in at least one way: type errors arising from such programs can be very difficult to debug.

- Abstraction over type classes. Abstraction over type classes is emulated by means of explicit dictionaries that represent the class that is being abstracted. This is an advanced type class programming technique and it might confuse beginning generic programmers.

- Arity based representations. Arity based representations are used to represent type constructors. However, it is more difficult to relate the type signature of an arity-based generic function to that of an instance. For example, generic map has type Rep2 $a b \rightarrow a \rightarrow b$, which does not bear a strong resemblance to the type of the BinTree instance of map: $(a \rightarrow b) \rightarrow$ BinTree $a \rightarrow$ BinTree $b$. For this reason, programming with arity-based representations might be challenging to a beginner.

The approaches that suffer from the first difficulty are SYB and SYB3. The second difficulty affects Smash. The third difficulty affects SYB3 and RepLib. The fourth difficulty affects LIGD, EMGM, and RepLib. However, the first two libraries, need such arities only occasionally, namely to define functions such as gmap and crushRight.

Another problem that can impede learning and use of an approach is the use of a relatively large number of implementation mechanisms. This is the case of SYB3 and RepLib. While it is possible to work out how one of the many mechanisms, for example GADTs in RepLib, is used into writing a generic function, it is much more difficult to understand the interactions of all the mechanisms in the same library. This, we believe, will make it more difficult for new users to learn and use SYB3 and RepLib.

\section{Conclusions}

We have introduced a set of criteria to compare libraries for generic programming in Haskell. These criteria can be viewed as a characterisation of generic programming in Haskell. Furthermore, we have designed a generic programming benchmark: a set of characteristic examples that check whether or not criteria are supported by generic programming libraries. Using the criteria and the benchmark, we have compared nine approaches to generic programming in Haskell.
Acknowledgements. This research has been partially funded by the Netherlands Organisation for Scientific Research (NWO), via the Real-life Datatype-Generic programming project, project nr. 612.063.613. We thank Jeremy Gibbons, Sean Leather and José Pedro Magalhães for their thoughtful comments and suggestions. We also thank the participants of the generics mailing list for the discussions and the code examples that sparked the work for this paper.

\section{References}

Frank Atanassow and Johan Jeuring. Inferring type isomorphisms generically. In MPC'04, volume 3125 of LNCS, pages 32-53. Springer, 2004.

Richard Bird and Lambert Meertens. Nested datatypes. In Johan Jeuring, editor, $M P C^{\prime} 98$, volume 1422 of $L N C S$, pages 52-67. Springer, 1998.

Björn Bringert and Aarne Ranta. A pattern for almost compositional functions. In ICFP'06, pages 216-226. ACM, 2006.

James Cheney and Ralf Hinze. A lightweight implementation of generics and dynamics. In Manuel Chakravarty, editor, Haskell'02, pages 90-104. ACM, 2002. doi: 10.1145/581690. 581698.

Koen Claessen and John Hughes. QuickCheck: A Lightweight Tool for Random Testing of Haskell Programs. In ICFP'00, pages 286-279. ACM, 2000.

Dave Clarke and Andres Löh. Generic haskell, specifically. In Proceedings of the IFIP TC2/WG2.1 Working Conference on Generic Programming, pages 21-47. Kluwer, B.V., 2003.

John Derrick and Simon Thompson. FORSE: Formally-Based Tool Support for Erlang Development. Project description, 2005. URL http://www.cs.kent.ac.uk/projects/forse/.

Ronald Garcia, Jaakko Järvi, Andrew Lumsdaine, Jeremy Siek, and Jeremiah Willcock. An extended comparative study of language support for generic programming. J. Funct. Program., 17(2): 145-205, 2007. doi: 10.1017/S0956796806006198.

The Haskell Generic Library list. Generic programming criteria template, 2008. Wiki page at haskell.org/haskellwiki/ Applications_and_libraries/Generic_programming/ Template.

The Haskell Prime list. Haskell prime, 2006. Wiki page at http: //hackage.haskell.org/trac/haskell-prime.

Ralf Hinze. Generics for the masses. Journal of Functional Programming, 16:451-482, 2006.

Ralf Hinze. Polytypic values possess polykinded types. Science of Computer Programming, 43(2-3):129-159, 2002.

Ralf Hinze and Andres Löh. "Scrap Your Boilerplate" revolutions. In Tarmo Uustalu, editor, $M P C^{\prime}$ '06, volume 4014 of $L N C S$, pages 180-208. Springer, 2006.

Ralf Hinze and Andres Löh. Generic programming, now! In Roland Backhouse et al., editors, Datatype-Generic Programming, LNCS, pages 150-208. Springer, 2007.

Ralf Hinze, Andres Löh, and Bruno C. d. S. Oliveira. "Scrap Your Boilerplate" reloaded. In Philip Wadler and Masimi Hagiya, editors, FLOPS'06, volume 3945 of LNCS. Springer, 2006.

Ralf Hinze, Johan Jeuring, and Andres Löh. Comparing approches to generic programming in haskell. In Roland Backhouse et al., editors, Datatype-Generic Programming, volume 4719 of LNCS, pages 72-149. Springer, 2007. doi: 10.1007/ 978-3-540-76786-2_2. 
Stefan Holdermans, Johan Jeuring, Andres Löh, and Alexey Rodriguez. Generic views on data types. In Tarmo Uustalu, editor, MPC'06, volume 4014 of $L N C S$, pages 209-234. Springer, 2006.

Paul Hudak, John Hughes, Simon Peyton Jones, and Philip Wadler. A history of Haskell: being lazy with class. In HOPL III, pages 12-1-12-55. ACM, 2007. doi: 10.1145/1238844.1238856.

Patrik Jansson and Johan Jeuring. PolyP - a polytypic programming language extension. In POPL'97, pages 470-482. ACM, 1997. doi: 10.1145/263699.263763.

Patrik Jansson and Johan Jeuring. Polytypic data conversion programs. Science of Computer Programming, 43(1):35-75, 2002.

Patrik Jansson and Johan Jeuring. PolyLib - a polytypic function library. Workshop on Generic Programming, Marstrand, June 1998.

Oleg Kiselyov. Smash your boilerplate without class and typeable. http://article.gmane.org/gmane.comp.lang. haskell.general/14086, 2006.

Oleg Kiselyov, Ralf Lammel, and Keean Schupke. Strongly typed heterogeneous collections. In Haskell '04: Proceedings of the ACM SIGPLAN workshop on Haskell, pages 96-107, New York, NY, USA, 2004. ACM Press. ISBN 1581138504. doi: 10.1145/1017472.1017488. URL http://portal.acm.org/ citation. cfm?id=1017488.

Pieter Koopman, Artem Alimarine, Jan Tretmans, and Rinus Plasmeijer. Gast: Generic automated software testing. In R. Peña and T. Arts, editors, IFL'02, volume 2670 of LNCS. Springer, 2003.

Ralf Lämmel and Simon Peyton Jones. Scrap your boilerplate: a practical approach to generic programming. ACM SIGPLAN Notices, 38(3):26-37, 2003. TLDI'03.

Ralf Lämmel and Simon Peyton Jones. Scrap more boilerplate: reflection, zips, and generalised casts. In ICFP'04, pages 244 255. ACM, 2004.

Ralf Lämmel and Simon Peyton Jones. Scrap your boilerplate with class: extensible generic functions. In ICFP'05, pages 204-215, 2005.

Ralf Lämmel and Joost Visser. A Strafunski Application Letter. In V. Dahl and P. Wadler, editors, $P A D L^{\prime} 03$, volume 2562 of $L N C S$, pages 357-375, 2003.

Ralf Lämmel and Joost Visser. Typed combinators for generic traversal. In PADL'02, volume 2257 of $L N C S$, pages 137-154. Springer, 2002.

Huiqing Li, Claus Reinke, and Simon Thompson. Tool support for refactoring functional programs. In Haskell'03, pages 27-38. ACM, 2003. doi: 10.1145/871895.871899.

Andres Löh, Dave Clarke, and Johan Jeuring. Depency-style Generic Haskell. In Olin Shivers, editor, ICFP'03, pages 141152, 2003.

Ian Lynagh. Template Haskell: A report from the field. URL: http://web.comlab.ox.ac.uk/oucl/work/ian.lynagh/ papers/, May 2003.

Lambert Meertens. Calculate polytypically! In H. Kuchen and S. D. Swierstra, editors, Programming Language Implementation and Logic Programming, volume 1140 of Lecture Notes in Computer Science, pages 1-16, 1996.

Neil Mitchell and Colin Runciman. A static checker for safe pattern matching in Haskell. In Trends in Functional Programming, volume 6. Intellect, 2007a. ISBN 9781841501765.

Neil Mitchell and Colin Runciman. Uniform boilerplate and list processing. In Haskell'07. ACM, 2007b.

Matthew Naylor and Colin Runciman. Finding inputs that reach a target expression. In $S C A M^{\prime} 07$, pages 133-142. IEEE Computer Society, 2007. doi: 10.1109/SCAM.2007.11.

Ulf Norell and Patrik Jansson. Polytypic programming in Haskell. In Phil Trinder et al., editors, IFL'03, volume 3145 of LNCS, pages 168-184. Springer, 2004.

Bruno C. d. S. Oliveira, Ralf Hinze, and Andres Löh. Extensible and modular generics for the masses. In Henrik Nilsson, editor, Trends in Functional Programming, pages 199-216, 2006.

Simon Peyton Jones, Dimitrios Vytiniotis, Stephanie Weirich, and Geoffrey Washburn. Simple unification-based type inference for GADTs. In ICFP'06, pages 50-61, 2006.

Philip Wadler. Theorems for free! In FPCA'89, pages 347-359. ACM, 1989.

Stephanie Weirich. RepLib: a library for derivable type classes. In Haskell'06, pages 1-12. ACM, 2006. doi: 10.1145/1159842. 1159844.

Noel Winstanley and John Meacham. DrIFT user guide, 2006. http://repetae.net/ john/computer/haskell/DrIFT/. 